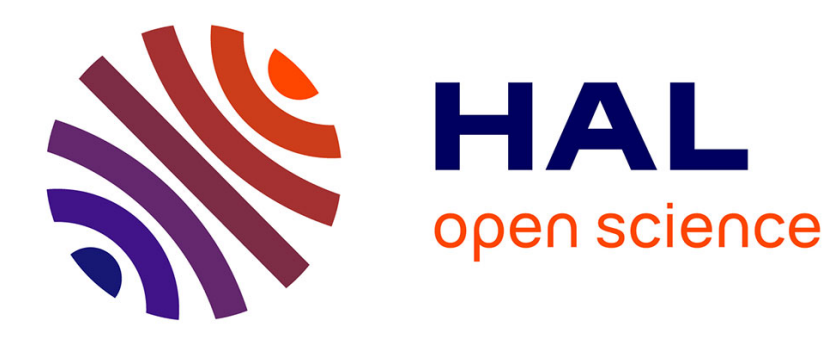

\title{
Optimal Control under Stochastic Target Constraints
}

\author{
Bruno Bouchard, Romuald Elie, Cyril Imbert
}

\section{To cite this version:}

Bruno Bouchard, Romuald Elie, Cyril Imbert. Optimal Control under Stochastic Target Constraints.

SIAM Journal on Control and Optimization, 2010, 48 (5), pp.3501-3531. hal-00373306

\section{HAL Id: hal-00373306 \\ https://hal.science/hal-00373306}

Submitted on 3 Apr 2009

HAL is a multi-disciplinary open access archive for the deposit and dissemination of scientific research documents, whether they are published or not. The documents may come from teaching and research institutions in France or abroad, or from public or private research centers.
L'archive ouverte pluridisciplinaire HAL, est destinée au dépôt et à la diffusion de documents scientifiques de niveau recherche, publiés ou non, émanant des établissements d'enseignement et de recherche français ou étrangers, des laboratoires publics ou privés. 


\title{
Optimal Control under Stochastic Target Constraints
}

\author{
Bruno Bouchard \\ CEREMADE, Université Paris Dauphine \\ and CREST-ENSAE \\ bouchard@ceremade.dauphine.fr
}

\author{
Romuald Elie \\ CEREMADE, Université Paris Dauphine \\ and CREST-ENSAE \\ elie@ceremade.dauphine.fr
}

\author{
Cyril Imbert \\ CEREMADE, Université Paris Dauphine \\ imbert@ceremade.dauphine.fr
}

March 2009

\begin{abstract}
We study a class of Markovian optimal stochastic control problems in which the controlled process $Z^{\nu}$ is constrained to satisfy an a.s. constraint $Z^{\nu}(T) \in G \subset \mathbb{R}^{d+1} \mathbb{P}$ - a.s. at some final time $T>0$. When the set is of the form $G:=\left\{(x, y) \in \mathbb{R}^{d} \times \mathbb{R}: g(x, y) \geq 0\right\}$, with $g$ non-decreasing in $y$, we provide a Hamilton-Jacobi-Bellman characterization of the associated value function. It gives rise to a state constraint problem where the constraint can be expressed in terms of an auxiliary value function $w$ which characterizes the set $D:=\left\{\left(t, Z^{\nu}(t)\right) \in\right.$ $[0, T] \times \mathbb{R}^{d+1}: Z^{\nu}(T) \in G$ a.s. for some $\left.\nu\right\}$. Contrary to standard state constraint problems, the domain $D$ is not given a-priori and we do not need to impose conditions on its boundary. It is naturally incorporated in the auxiliary value function $w$ which is itself a viscosity solution of a non-linear parabolic PDE. Applying ideas recently developed in Bouchard, Elie and Touzi (2008), our general result also allows to consider optimal control problems with moment constraints of the form $\mathbb{E}\left[g\left(Z^{\nu}(T)\right)\right] \geq 0$ or $\mathbb{P}\left[g\left(Z^{\nu}(T)\right) \geq 0\right] \geq p$.
\end{abstract}

Key words: Optimal control, State constraint problems, Stochastic target problem, discontinuous viscosity solutions.

Mathematical subject classifications: Primary 93E20, 49L25; secondary 60J60. 


\section{Introduction}

The aim of this paper is to study stochastic control problems under stochastic target constraint of the form

$$
V(t, z):=\sup \left\{\mathbb{E}\left[f\left(Z_{t, z}^{\nu}(T)\right)\right], \nu \in \mathcal{U} \text { s.t. } g\left(Z_{t, z}^{\nu}(T)\right) \geq 0 \mathbb{P}-\text { a.s. }\right\}
$$

where the controlled process $Z_{t, z}^{\nu}$ is the strong solution of a stochastic differential equation,

$$
Z_{t, z}^{\nu}(s)=z+\int_{t}^{s} \mu_{Z}\left(Z_{t, z}^{\nu}(r), \nu_{r}\right) d r+\int_{t}^{s} \sigma_{Z}\left(Z_{t, z}^{\nu}(r), \nu_{r}\right) d W_{r}, t \leq s \leq T
$$

for some $d$-dimensional Brownian motion $W$, and the set of controls $\mathcal{U}$ is the collection of $U$-valued square integrable progressively measurable processes (for the filtration generated by $W$ ), for some closed set $U \subset \mathbb{R}^{d}$.

Such problems naturally appear in economics where a controller tries to maximize (or minimize) a criteria under some a.s. constraint on the final output. It is typically the case in finance or insurance where a manager tries to maximize the utility (or minimize the expected value of a loss function) of the terminal value of his portfolio under some strong no-bankruptcy type condition, see e.g. [16], [7] and the references therein. See also the example of application in Section 4 below.

From the mathematical point of view, (1.1) can be seen as a state constraint problem. However, it is at first glance somehow "non-standard" because the a.s. constraint is imposed only at $t=T$ and not on $[0, T]$ as usual, compare with [9], [13], [14], [17] and [18].

Our first observation is that it can actually be converted into a "classical" state constraint problem:

$$
V(t, z)=\sup \left\{\mathbb{E}\left[f\left(Z_{t, z}^{\nu}(T)\right)\right], \nu \in \mathcal{U} \text { s.t. } Z_{t, z}^{\nu}(s) \in D \mathbb{P}-\text { a.s. for all } t \leq s \leq T\right\}
$$

where

$$
D:=\left\{(t, z) \in[0, T] \times \mathbb{R}^{d+1}: g\left(Z_{t, z}^{\nu}(T)\right) \geq 0 \mathbb{P}-\text { a.s. for some } \nu \in \mathcal{U}\right\}
$$

is the corresponding viability domain, in the terminology of [1]. This is indeed an immediate consequence of the so-called geometric dynamic programming principle introduced by Soner and Touzi [21] and [22] in the context of stochastic target problems:

$$
g\left(Z_{t, z}^{\nu}(T)\right) \geq 0 \mathbb{P}-\text { a.s. } \Longleftrightarrow\left(s, Z_{t, z}^{\nu}(s)\right) \in D \mathbb{P}-\text { a.s. for all } t \leq s \leq T \text {. }
$$

There exists a huge literature on state constraint problems. For deterministic control problems, Soner [20] first derived the Bellman equation under the appropriate form in order to characterize the value function as the unique viscosity solution. The main issue is to deal with the boundary of the domain. One of the important contribution of [20] is to show that the value function is the unique viscosity solution of the Bellman equation which is a subsolution "up to the boundary". Hence, loosely speaking, Soner exhibited a boundary condition ensuring the uniqueness of the viscosity solution. A rather complete study for first order equations can be found in [9]. See 
also [13]. For stochastic control problems, the study of viscosity solutions with such boundary condition was initiated in [18] where the volatility is the identity matrix. The results of [18] have been generalized since then, see for instance $[17,2,14]$.

The main difference with standard state constraint problems is that, in this paper, the set $D$ is not given a-priori but is defined implicitly by a stochastic target problem. We should also emphasize the fact that we focus here on the derivation of the PDEs and not on the study of the PDEs in themselves (comparison, existence of regular solutions, etc...). The last point, which is obviously very important, is left for further research.

In this paper, we shall restrict to the case where $(x, y) \in \mathbb{R}^{d} \times \mathbb{R} \mapsto g(x, y)$ is non-decreasing in $y$, and $Z_{t, z}^{\nu}$ is of the form $\left(X_{t, x}^{\nu}, Y_{t, x, y}^{\nu}\right)$, where $\left(X_{t, x}^{\nu}, Y_{t, x, y}^{\nu}\right)$ takes values in $\mathbb{R}^{d} \times \mathbb{R}$ and follows the dynamics

$$
\begin{aligned}
X_{t, x}^{\nu}(s) & =x+\int_{t}^{s} \mu_{X}\left(X_{t, x}^{\nu}(r), \nu_{r}\right) d r+\int_{t}^{s} \sigma_{X}\left(X_{t, x}^{\nu}(r), \nu_{r}\right) d W_{r} \\
Y_{t, x, y}^{\nu}(s) & =y+\int_{t}^{s} \mu_{Y}\left(Z_{t, x, y}^{\nu}(r), \nu_{r}\right) d r+\int_{t}^{s} \sigma_{Y}\left(Z_{t, x, y}^{\nu}(r), \nu_{r}\right)^{\top} d W_{r} .
\end{aligned}
$$

In this case, the set $\{y \in \mathbb{R}:(t, x, y) \in D\}$ is a half-space, for fixed $(t, x)$. This allows to characterize the (closure of the) set $D$ in terms of the auxiliary value function

$$
w(t, x):=\inf \{y \in \mathbb{R}:(t, x, y) \in D\} .
$$

Assuming that the inf is always achieved in the definition of $w,(1.2)$ can then be written equivalently as

$V(t, x, y)=\sup \left\{\mathbb{E}\left[f\left(Z_{t, x, y}^{\nu}(T)\right)\right], \nu \in \mathcal{U}\right.$ s.t. $Y_{t, x, y}^{\nu}(s) \geq w\left(s, X_{t, x}^{\nu}(s)\right) \mathbb{P}-$ a.s. for all $\left.t \leq s \leq T\right\}$

We should immediately observe that more general situations could be discussed by following the approach of [21] which consists in replacing $w$ by $\bar{w}(t, z):=1-\mathbf{1}_{(t, z) \in D}$, and working with $\bar{w}$ instead of $w$.

As usual in state constraints problems, the constraint is not binding until one reaches the boundary. We actually show that $V$ solves (in the discontinuous viscosity sense) the usual Hamilton-JacobiBellman equation

$$
-\sup _{u \in U} \mathcal{L}_{Z}^{u} V=0 \quad \text { where } \quad \mathcal{L}_{Z}^{u} V:=\partial_{t} V+\left\langle\mu_{Z}(\cdot, u), D V\right\rangle+\frac{1}{2} \operatorname{Tr}\left[\sigma_{Z} \sigma_{Z}^{\top}(\cdot, u) D^{2} V\right],
$$

in the interior of the domain $D$ with the appropriate boundary condition at $t=T$

$$
V(T-, \cdot)=f
$$

On the other hand, assuming for a while that $w$ is smooth (and that the inf is always achieved in its definition), any admissible control $\nu$ should be such that $d Y_{t, x, y}^{\nu}(s) \geq d w\left(s, X_{t, x}^{\nu}(s)\right)$ when 
$\left(s, X_{t, x}^{\nu}(s), Y_{t, x, y}^{\nu}(s)\right) \in \partial_{Z} D:=\left\{(t, x, y) \in[0, T) \times \mathbb{R}^{d} \times \mathbb{R}: y=w(t, x)\right\}$, in order to avoid to cross the boundary of $D$. Applying Itô's Lemma to $w$, this shows that $\nu_{s}$ should satisfy:

$$
\sigma_{Y}\left(Z_{t, x, y}^{\nu}(s), \nu_{s}\right)=\sigma_{X}\left(X_{t, x}^{\nu}(s), \nu_{s}\right)^{\top} D w\left(s, X_{t, x}^{\nu}(s)\right) \quad \text { and } \quad \mu_{Y}\left(Z_{t, x, y}^{\nu}(s), \nu_{s}\right) \geq \mathcal{L}_{X}^{\nu_{s}} w\left(s, X_{t, x}^{\nu}(s)\right)
$$

where $\mathcal{L}_{X}^{u}$ denotes the Dynkin operator of $X$ for the control $u$. This formally shows that $V$ solves the constrained Hamilton-Jacobi-Bellman equation

$$
-\sup _{u \in U(t, x, y)} \mathcal{L}_{Z}^{u} V=0 \text { where } U(\cdot):=\left\{u \in U: \sigma_{Y}(\cdot, u)=\sigma_{X}(\cdot, u)^{\top} D w, \mu_{Y}(\cdot, u)-\mathcal{L}_{X}^{u} w \geq 0\right\}
$$

on the boundary $\partial_{Z} D$, where, by Soner and Touzi [22] and Bouchard, Elie and Touzi [5], the auxiliary value function $w$ is a (discontinuous viscosity) solution of

$$
\sup _{u \in U, \sigma_{Y}(\cdot, u)=\sigma_{X}(\cdot, u)^{\top} D w}\left(\mu_{Y}(\cdot, w, u)-\mathcal{L}_{X}^{u} w\right)=0 \quad, \quad g(\cdot, w(T-, \cdot))=0 .
$$

This implies that no a-priori assumption has to be imposed on the boundary on $D$ and the coefficients $\mu_{Z}$ and $\sigma_{Z}$ in order to ensure that $Z_{t, z}^{\nu}$ can actually be reflected on the boundary. It is automatically incorporated in the auxiliary value function through its PDE characterization (1.7). This is also a main difference with the literature on state constraints where either strong conditions are imposed on the boundary, so as to insure that the constrained process can be reflected, or, the solution explodes on the boundary, see the above quoted papers.

From the numerical point of view, the characterization (1.7) also allows to compute the value function $V$ by first solving (1.7) to compute $w$ and then using $w$ to solve (1.4)-(1.5)-(1.6).

The main difficulties come from the following points:

1. The stochastic control problem (1.1) being non-standard, we first need to establish a dynamic programming principle for optimal control under stochastic constraints. This is done by appealing to the geometric dynamic principle of Soner and Touzi [21].

2. The auxiliary value function $w$ is in general not smooth. To give a sense to (1.6), we therefore need to consider a weak formulation which relies on test functions for $w$. This essentially amounts to consider proximal normal vector at the singular points of $\partial D$, see [10].

3. The set $U$ in which the controls take their values is a-priori not compact, which makes the equations (1.4) and (1.6) discontinuous. As usual, this is overcome by considering the lower- and upper-semicontinuous envelopes of the corresponding operators, see [11].

We should also note that, in classical state constraint problems, the subsolution property on the spacial boundary is usually not fully specified, see the above quoted papers. One can typically only show that the PDE (1.4) propagates up to the boundary. In the case where $w$ is continuous, we actually prove that $V$ is a subsolution of the constraint Hamilton-Jacobi-Bellman equation (1.6) on $\partial_{Z} D$. Under additional assumptions on the coefficients and $w$, this allows to provide a PDE characterization for $\mathcal{V}(t, x):=V(t, x, w(t, x))$ and allows to replace the boundary condition (1.6) by a simple Dirichlet condition $V(t, x, y)=\mathcal{V}(t, x)$ on $\{y=w(t, x)\}$. This simplifies the numerical 
resolution of the problem: first solve (1.7) to compute $w$ and therefore $D$, then solve the PDE associated to $\mathcal{V}$, finally solve the Hamilton-Jacobi-Bellman equation (1.4) in $D$ with the boundary conditions (1.5) at $t=T$ and $V(t, x, y)=\mathcal{V}(t, x)$ on $\partial_{Z} D$.

Many extensions of this work could be considered. First, jumps could be introduced without much difficulties, see [4] and [19] for stochastic target problems with jumps. One could also consider more stringent conditions of the form $g\left(Z_{t, z}^{\nu}(s)\right) \geq 0 \mathbb{P}-$ a.s. for all $t \leq s \leq T$ by applying the American version of the geometric dynamic programming of [6], or moments constraints of the form $\mathbb{E}\left[g\left(Z_{t, z}^{\nu}(T)\right)\right] \geq 0 \mathbb{P}$ - a.s. or $\mathbb{P}\left[g\left(Z_{t, z}^{\nu}(T)\right) \geq 0\right] \geq p$ by following the ideas introduced in [5]. Such extension are rather immediate and will be discussed in Section 5 below.

The rest of the paper is organized as follows. The problem and its link with standard state constraints problems are presented in Section 2. Section 3 contains our main results. The possible immediate extensions and an example of application are discussed in Section 5 and Section 4 . The proofs are collected in Section 6. Finally, the Appendix contains the proof of a version of the geometric dynamic programming principle we shall use in this paper.

Notations: For any $\kappa \in \mathbb{N}$, we shall use the following notations. Any element of $\mathbb{R}^{\kappa}$ is viewed as a column vector. The Euclidean norm of a vector or a matrix is denoted by $|\cdot|,{ }^{\top}$ stands for transposition, and $\langle\cdot, \cdot\rangle$ denotes the natural scalar product. We denote by $\mathbb{M}^{\kappa}$ (resp. $\mathbb{S}^{\kappa}$ ), the set of $\kappa$-dimensional (resp. symmetric) square matrices. Given a smooth function $\varphi:(t, x) \in \mathbb{R}_{+} \times \mathbb{R}^{\kappa} \mapsto$ $\varphi(t, x) \in \mathbb{R}$, we denote by $\partial_{t} \varphi$ its derivative with respect to its first variable, and by $D \varphi$ and $D^{2} \varphi$ its Jacobian and Hessian matrix with respect to the second one. For a set $\mathcal{O} \subset \mathbb{R}^{\kappa}$, int $(\mathcal{O})$ denotes its interior, $\operatorname{cl}(\mathcal{O})$ its closure and $\partial \mathcal{O}$ its boundary. If $B=[s, t] \times \mathcal{O}$ for $s \leq t$ and $\mathcal{O} \subset \mathbb{R}^{\kappa}$, we write $\partial_{p} B:=([s, t) \times \partial \mathcal{O}) \cup(\{t\} \times \operatorname{cl}(\mathcal{O}))$ for its parabolic boundary. Given $r>0$ and $x \in \mathbb{R}^{\kappa}, B_{r}(x)$ denotes the open ball of radius $r$ and center $x$. In the following, the variable $z \in \mathbb{R}^{d+1}$ will often be understood as a couple $(x, y) \in \mathbb{R}^{d} \times \mathbb{R}$.

\section{The optimal control problem under stochastic target constraint}

\subsection{Problem formulation}

Let $T>0$ be the finite time horizon and let $\Omega$ denote the space of $\mathbb{R}^{d}$-valued continuous functions $\left(\omega_{t}\right)_{t \leq T}$ on $[0, T], d \geq 1$, endowed with the Wiener measure $\mathbb{P}$. We denote by $W$ the coordinate mapping, i.e. $\left(W(\omega)_{t}\right)_{t \leq T}=\left(\omega_{t}\right)_{t \leq T}$ for $\omega \in \Omega$, so that $W$ is a $d$-dimensional Brownian motion on the canonical filtered probability space $(\Omega, \mathcal{F}, \mathbb{P}, \mathbb{F})$ where $\mathcal{F}$ is the Borel tribe of $\Omega$ and $\mathbb{F}=$ $\left\{\mathcal{F}_{t}, 0 \leq t \leq T\right\}$ is the $\mathbb{P}$-augmentation of the right-continuous filtration generated by $W$.

Let $\mathcal{U}$ be the collection of progressively measurable processes $\nu$ in $L^{2}([0, T] \times \Omega)$, with values in a given closed subset $U$ of $\mathbb{R}^{d}$.

For $t \in[0, T], z=(x, y) \in \mathbb{R}^{d} \times \mathbb{R}$ and $\nu \in \mathcal{U}$, the controlled process $Z_{t, z}^{\nu}:=\left(X_{t, x}^{\nu}, Y_{t, x, y}^{\nu}\right)$ is defined as the $\mathbb{R}^{d} \times \mathbb{R}$-valued unique strong solution of the stochastic differential equation (1.3) where $\left(\mu_{X}, \sigma_{X}\right):(x, u) \in \mathbb{R}^{d} \times U \mapsto \mathbb{R}^{d} \times \mathbb{M}^{d}$ and $\left(\mu_{Y}, \sigma_{Y}\right):(z, u) \in \mathbb{R}^{d} \times \mathbb{R} \times U \mapsto \mathbb{R} \times \mathbb{R}^{d}$ are assumed 
to be Lipschitz continuous. Note that this implies that $\sup _{t \leq s \leq T}\left|Z_{t, z}^{\nu}\right|$ is bounded in $L^{2}(\Omega)$, for any $\nu \in \mathcal{U}$.

For later use, it is convenient to consider $\mu_{Z}: \mathbb{R}^{d+1} \times U \longrightarrow \mathbb{R}^{d+1}$ and $\sigma_{Z}: \mathbb{R}^{d+1} \times U \longrightarrow \mathbb{M}^{d+1, d}$ defined, for $z=(x, y) \in \mathbb{R}^{d+1}$, as

$$
\mu_{Z}(z, u):=\left(\begin{array}{c}
\mu_{X}(x, u) \\
\mu_{Y}(z, u)
\end{array}\right) \quad \sigma_{Z}(z, u):=\left[\begin{array}{c}
\sigma_{X}(x, u) \\
\sigma_{Y}(z, u)^{\top}
\end{array}\right] .
$$

The aim of this paper is to provide a PDE characterization of the value function of the optimal control problem under stochastic target constraint

$$
(t, z) \mapsto \sup \left\{\mathbb{E}\left[f\left(Z_{t, z}^{\nu}(T)\right)\right], \nu \in \mathcal{U} \text { s.t. } g\left(Z_{t, z}^{\nu}(T)\right) \geq 0 \mathbb{P}-\text { a.s. }\right\}
$$

which means that we restrict ourselves to controls satisfying the stochastic target constraints $g\left(Z_{t, z}^{\nu}(T)\right) \geq 0 \mathbb{P}-$ a.s.

In order to give a sense to the above expression, we shall assume all over this paper that $f, g$ : $\mathbb{R}^{d+1} \rightarrow \mathbb{R}$ are two locally bounded Borel-measurable maps, and that $f$ has quadratic growth, which ensures that the above expectation is well defined for any $\nu \in \mathcal{U}$.

For technical reasons related to the proof of the dynamic programming principle (see Section 6.1 and the Appendix below), we need to restrict ourselves at time $t$ to the subset $\mathcal{U}^{t}$ of controls defined as follows

$$
\mathcal{U}^{t}:=\left\{\nu \in \mathcal{U}: \nu \text { independent of } \mathcal{F}_{t}\right\}
$$

Our problem is thus formulated as

$$
V(t, z):=\sup _{\nu \in \mathcal{U}_{t, z}} J(t, z ; \nu)
$$

where

$$
\begin{aligned}
J(t, z ; \nu) & :=\mathbb{E}\left[f\left(Z_{t, z}^{\nu}(T)\right)\right] \text { for } \nu \in \mathcal{U} \\
\mathcal{U}_{t, z} & :=\left\{\nu \in \mathcal{U}^{t}: g\left(Z_{t, z}^{\nu}(T)\right) \geq 0 \mathbb{P} \text { - a.s. }\right\}
\end{aligned}
$$

For sake of simplicity, we shall only consider the case where, for every fixed $x$,

$$
y \longmapsto g(x, y) \quad \text { is non-decreasing and right-continuous. }
$$

Remark 2.1. It follows from Assumption (2.2) that $\mathcal{U}_{t, x, y} \supset \mathcal{U}_{t, x, y^{\prime}}$ for $y \geq y^{\prime}$.

Remark 2.2. We have imposed above strong Lipschitz continuity assumptions and integrability conditions on the coefficients and the set of controls in order to avoid additional technical difficulties. It will be clear from the proofs that these conditions can be relaxed in particular situations whenever the quantities introduced below are well defined. 


\subsection{Dynamic programming for stochastic targets problem and interpretation as a state constraint problem}

Note that the above problem is well-posed only on the domain

$$
D:=\left\{(t, z) \in[0, T] \times \mathbb{R}^{d+1}: \mathcal{U}_{t, z} \neq \emptyset\right\},
$$

and that, at least formally, the control problem (2.1) is equivalent to the state constraint problem

$$
\sup \left\{\mathbb{E}\left[f\left(Z_{t, z}^{\nu}(T)\right)\right], \nu \in \mathcal{U} \text { s.t. } Z_{t, z}^{\nu}(s) \in D \mathbb{P}-\text { a.s. } \forall t \leq s \leq T\right\}
$$

This can be made rigorous by appealing to the geometric dynamic programming principle of Soner and Touzi [21].

Theorem 2.1. For any $(t, z) \in[0, T) \times \mathbb{R}^{d+1}$ and $\nu \in \mathcal{U}^{t}$, we have

$$
\exists \tilde{\nu} \in \mathcal{U}_{t, z} \text { s.t. } \nu=\tilde{\nu} \text { on }[t, \theta) \Longleftrightarrow\left(\theta, Z_{t, z}^{\nu}(\theta)\right) \in D \mathbb{P}-\text { a.s. }
$$

for all $[t, T]$-valued stopping time $\theta$.

The proof of this result is provided in [21] when the set of controls is the whole set $\mathcal{U}$ instead of $\mathcal{U}^{t}$. We explain in the Appendix how to modify their arguments in our framework.

This result can be reformulated in terms of the auxiliary value function $w$ defined as follows

$$
w(t, x):=\inf \{y \in \mathbb{R}:(t, x, y) \in D\}
$$

which, by Remark 2.1, characterizes (the closure of) $D$.

Corollary 2.1. For any $(t, x, y) \in[0, T) \times \mathbb{R}^{d} \times \mathbb{R}, \nu \in \mathcal{U}^{t}$ and $[t, T]$-valued stopping time $\theta$, we have:

1. If $Y_{t, x, y}^{\nu}(\theta)>w\left(\theta, X_{t, x}^{\nu}(\theta)\right) \mathbb{P}-$ a.s., then there exists a control $\tilde{\nu} \in \mathcal{U}_{t, x, y}$ such that $\nu=\tilde{\nu}$ on $[t, \theta)$.

2. If there exists $\tilde{\nu} \in \mathcal{U}_{t, x, y}$ such that $\nu=\tilde{\nu}$ on $[t, \theta)$, then $Y_{t, x, y}^{\nu}(\theta) \geq w\left(\theta, X_{t, x}^{\nu}(\theta)\right) \mathbb{P}-$ a.s.

Note that the later result allows us to provide a PDE characterization of the auxiliary value function, see (1.7). A rigorous version is reported in Theorem 3.2 below under strong smoothness assumptions. The non-smooth case is studied in [22] when $U$ is bounded and in [5] when $U$ is not bounded, and we refer to these papers for the exact formulation in more general situations.

From now on, we shall work under the following

Standing assumption: $w$ is continuous on $[0, T) \times \mathbb{R}^{d}$ and admits a continuous extension $\hat{w}$ on $[0, T] \times \mathbb{R}^{d}$ such that $g(\cdot, \hat{w}(T, \cdot)) \geq 0$ on $\mathbb{R}^{d}$.

In the following, we shall write $w(T, \cdot)$ for $\hat{w}(T, \cdot)$ for ease of notations. 
Note that the previous assumption implies that $\operatorname{cl}(D)$ can be written $\operatorname{as} \operatorname{int}_{p}(D) \cup \partial_{p} D$ with

$$
\left\{\begin{aligned}
\operatorname{int}_{p}(D) & =\left\{(t, x, y) \in[0, T) \times \mathbb{R}^{d+1}: y>w(t, x)\right\} \\
\partial_{p} D & =\partial_{Z} D \cup \partial_{T} D
\end{aligned}\right.
$$

where

$$
\begin{aligned}
\partial_{Z} D & :=\partial D \cap\left([0, T) \times \mathbb{R}^{d+1}\right)=\left\{(t, x, y) \in[0, T) \times \mathbb{R}^{d+1}: y=w(t, x)\right\} \\
\partial_{T} D & :=\partial D \cap\left(\{T\} \times \mathbb{R}^{d+1}\right)=\left\{(t, x, y) \in\{T\} \times \mathbb{R}^{d+1}: y \geq w(t, x)\right\}
\end{aligned}
$$

\section{Viscosity characterization of the value function}

Before stating our main result, let us start with a formal discussion.

\subsection{Formal discussion}

In the interior of the domain. First, it follows from Theorem 2.1 that the constraint is not binding in the interior of the domain. We can thus expect $V$ to be a viscosity solution on $\operatorname{int}_{p}(D)$ of the usual Hamilton-Jacobi-Bellman equation

$$
-\partial_{t} \varphi(t, z)+H\left(z, D \varphi(t, z), D^{2} \varphi(t, z)\right)=0
$$

where, for $(z, q, A) \in \mathbb{R}^{d+1} \times \mathbb{R}^{d+1} \times \mathbb{S}^{d+1}$,

$$
\left\{\begin{aligned}
H(z, q, A) & :=\inf _{u \in U} H^{u}(z, q, A) \\
H^{u}(z, q, A) & :=-\left\langle\mu_{Z}(z, u), q\right\rangle-\frac{1}{2} \operatorname{Tr}\left[\left(\sigma_{Z} \sigma_{Z}^{\top}\right)(z, u) A\right] .
\end{aligned}\right.
$$

However, since $U$ may not be bounded, the above operator is not necessarily continuous and we shall have to relax it and consider its lower- and upper-semicontinuous envelopes $H_{*}$ and $H^{*}$ on $\mathbb{R}^{d} \times \mathbb{R} \times \mathbb{R}^{d+1} \times \mathbb{S}^{d+1}$.

On the time boundary. From the definition of $V$, one could also expect that $V_{*}(T, z) \geq f_{*}(z)$ and $V^{*}(T, z) \leq f^{*}(z)$. This will indeed be satisfied and the proof is actually standard. The only difficulty comes from the fact that $U$ may be unbounded.

On the spacial boundary. We now discuss the boundary condition on $\partial_{Z} D$. First note that Theorem 2.1 implies that the process $Z_{t, z}^{\nu}$ should never cross the boundary $\partial_{Z} D$ whenever $\nu \in \mathcal{U}_{t, z}$. In view of $(2.5)$, this implies that at the limit, when $y \rightarrow w(t, x)$, the process $Y_{t, x, y}^{\nu}-w\left(\cdot, X_{t, x}^{\nu}\right)$ should have a non-negative drift and a zero volatility. This means that, at a formal level, the control $\nu$ should satisfy

$$
\begin{array}{r}
\sigma_{Y}\left(x, y, \nu_{t}\right)-\sigma_{X}\left(x, \nu_{t}\right)^{\top} D w(t, x)=0 \\
\mu_{Y}\left(x, y, \nu_{t}\right)-\mathcal{L}_{X}^{\nu_{t}} w(t, x) \quad \geq 0
\end{array}
$$


where $\mathcal{L}_{X}^{u}$ denotes the Dynkin operator of $X$; precisely, for a smooth function $\varphi \in C^{1,2}$,

$$
\mathcal{L}_{X}^{u} \varphi(t, x):=\partial_{t} \varphi(t, x)+\left\langle\mu_{X}(x, u), D \varphi(t, x)\right\rangle+\frac{1}{2} \operatorname{Tr}\left[\left(\sigma_{X} \sigma_{X}^{\top}\right)(x, u) D^{2} \varphi(t, x)\right] .
$$

Hence, $V$ should satisfy on $\partial_{Z} D$ the following equation

$$
-\partial_{t} \varphi(t, z)+H_{\text {int }}\left(z, D \varphi(t, z), D^{2} \varphi(t, z)\right)=0
$$

where

$$
H_{\mathrm{int}}(t, x, y, q, A)=\inf _{u \in U_{\mathrm{int}}(t, x, y, w)} H^{u}(x, y, q, A)
$$

and

$$
U_{\text {int }}(t, x, y, w)=\left\{u \in U: \sigma_{Y}(x, y, u)-\sigma_{X}(x, u)^{\top} D w(t, x)=0, \mu_{Y}(x, y, u)-\mathcal{L}_{X}^{u} w(t, x) \geq 0\right\}
$$

corresponds to controls driving the process inside the domain. Remark that $H_{\mathrm{int}} \geq H$ since $U_{\text {int }} \subset U$.

Since $w$ may not be smooth, we need to use the notion of test functions to give a precise meaning to the above expression. We therefore introduce the set $\mathcal{W}^{*}(t, x)$ defined as follows

$$
\mathcal{W}^{*}(t, x)=\left\{\phi \in C^{1,2}\left([0, T] \times \mathbb{R}^{d}\right) \text { s.t. }(w-\phi)<(w-\phi)(t, x)=0 \text { in a neighborhood of }(t, x)\right\} .
$$

The set $\mathcal{W}_{*}(t, x)$ is defined analogously:

$$
\mathcal{W}_{*}(t, x)=\left\{\phi \in C^{1,2}\left([0, T] \times \mathbb{R}^{d}\right) \text { s.t. }(w-\phi)>(w-\phi)(t, x)=0 \text { in a neighborhood of }(t, x)\right\} .
$$

Our operators are then defined as follows:

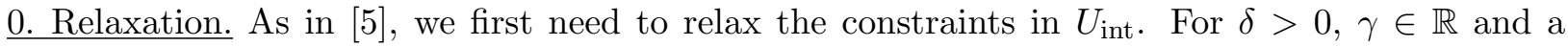
smooth function $\phi$, we therefore define

$$
\begin{aligned}
N^{u}(x, y, q) & :=\sigma_{Y}(x, y, u)-\sigma_{X}(x, u)^{\top} q \\
\mathcal{N}_{\delta}(x, y, q) & :=\left\{u \in U:\left|N^{u}(x, y, q)\right| \leq \delta\right\} \\
U_{\delta, \gamma}(t, x, y, \phi) & :=\left\{u \in \mathcal{N}_{\delta}(x, y, D \phi(t, x)): \mu_{Y}(x, y, u)-\mathcal{L}_{X}^{u} \phi(t, x) \geq \gamma\right\}
\end{aligned}
$$

and

$$
F_{\delta, \gamma}^{\phi}(t, z, q, A):=\inf _{u \in U_{\delta, \gamma}(t, z, \phi)}\left\{-\left\langle\mu_{Z}(z, u), q\right\rangle-\operatorname{Tr}\left[\left(\sigma_{Z} \sigma_{Z}^{\top}\right)(z, u) A\right]\right\}
$$

with the convention $\inf \emptyset=\infty$. The set $U_{\delta, \gamma}(\cdot, \phi)$ and the operator $F_{\delta, \gamma}^{\phi}$ will correspond to the relaxed versions of $U_{\text {int }}$ and $H_{\text {int }}$ associated to a test function $\phi$ for $w$.

Remark 3.1. Remark that the fact that $U_{\delta, \gamma}(t, z, \phi) \subset U$ implies in particular that, for all $(t, z, q, A)$, we have

$$
F_{\delta, \gamma}^{\phi}(t, z, q, A) \geq H(z, q, A)
$$

for any smooth function $\phi$. 
1. Supersolution. We first relax the supersolution property by classically considering the upper semi-relaxed limit $F^{\phi *}$ of the family of functions $\left(F_{\delta, \gamma}^{\phi}\right)_{\delta, \gamma}$. We recall that it is defined as follows

$$
F^{\phi *}(t, z, q, A):=\limsup _{\substack{\left(t^{\prime}, z^{\prime}, q^{\prime}, A^{\prime}\right) \rightarrow(t, z, q, A) \\\left(\delta^{\prime}, \gamma^{\prime}\right) \rightarrow 0}} F_{\delta^{\prime}, \gamma^{\prime}}^{\phi}\left(t^{\prime}, z^{\prime}, q^{\prime}, A^{\prime}\right),
$$

for $\phi \in \mathcal{W}^{*}(t, x)$ and $(t, z, q, A) \in[0, T] \times \mathbb{R}^{d} \times \mathbb{R}^{d+1} \times \mathbb{S}^{d+1}$.

Note that, when $w \in C^{1,2}, \phi \in \mathcal{W}^{*}(t, x)$ implies that $\partial_{t} w(t, x) \leq \partial_{t} \phi(t, x), D w(t, x)=D \phi(t, x)$ and $D^{2} w(t, x) \leq D^{2} \phi(t, x)$, which leads to $U_{\delta, \gamma}(\cdot, \phi) \subset U_{\delta, \gamma}(\cdot, w)$ and therefore

$$
\text { if } w \in C^{1,2} \text {, then } \forall \phi \in \mathcal{W}^{*}(t, x), \quad F_{\delta, \gamma}^{\phi}(t, z, q, A) \geq F_{\delta, \gamma}^{w}(t, z, q, A) .
$$

Also note that

$$
F^{\phi *}(t, z, q, A):=\limsup _{\substack{\left(t^{\prime}, z^{\prime}, q^{\prime}, A^{\prime}\right) \rightarrow(t, z, q, A) \\ \gamma^{\prime} \downarrow 0}} F_{0, \gamma^{\prime}}^{\phi}\left(t^{\prime}, z^{\prime}, q^{\prime}, A^{\prime}\right),
$$

since $U_{0, \gamma^{\prime}} \subset U_{\delta, \gamma}$ for $\delta \geq 0$ and $\gamma \leq \gamma^{\prime}$. Moreover, it follows from (3.2) that $F^{\phi *}$ is the uppersemicontinuous envelope of

$$
\overline{\mathcal{H}}^{\phi}(t, z, q, A):= \begin{cases}H^{*}(z, q, A) & \text { if }(t, z) \in \operatorname{int}_{p}(D) \\ F^{\phi *}(t, z, q, A) & \text { if }(t, z) \in \partial_{p} D\end{cases}
$$

on $\partial_{p} D$ which is consistent with Definition 7.4 in [11].

2. Subsolution. In order to relax the subsolution property, we consider the lower relaxed semi-limit $F_{*}^{\phi}$ of $\left(F_{\delta, \gamma}^{\phi}\right)_{\delta, \gamma}$. We recall that

$$
F_{*}^{\phi}(t, z, q, A):=\liminf _{\substack{\left(t^{\prime}, z^{\prime}, q^{\prime}, A^{\prime}\right) \rightarrow(t, z, q, A) \\\left(\delta^{\prime}, \gamma^{\prime}\right) \rightarrow 0}} F_{\delta^{\prime}, \gamma^{\prime}}^{\phi}\left(t^{\prime}, z^{\prime}, q^{\prime}, A^{\prime}\right)
$$

for $\phi \in \mathcal{W}^{*}(t, x)$ and $(t, z, q, A) \in[0, T] \times \mathbb{R}^{d} \times \mathbb{R}^{d+1} \times \mathbb{S}^{d+1}$.

As above, we can note that, when $w \in C^{1,2}, \phi \in \mathcal{W}_{*}(t, x)$ implies that $\partial_{t} w(t, x) \geq \partial_{t} \phi(t, x)$, $D w(t, x)=D \phi(t, x)$ and $D^{2} w(t, x) \geq D^{2} \phi(t, x)$, which leads to

$$
\text { if } w \in C^{1,2} \text {, then } \forall \phi \in \mathcal{W}_{*}(t, x), \quad F_{\delta, \gamma}^{\phi}(t, z, q, A) \leq F_{\delta, \gamma}^{w}(t, z, q, A) \text {. }
$$

However, (3.2) implies that $F_{*}^{\phi} \geq H_{*}$. Hence,

$$
\underline{\mathcal{H}}^{\phi}(z, q, A):= \begin{cases}H_{*}(z, q, A) & \text { if }(t, z) \in \operatorname{int}_{p}(D) \\ F_{*}^{\phi}(t, z, q, A) & \text { if }(t, z) \in \partial_{p} D\end{cases}
$$

may not be lower-semicontinuous. Since, according to [11], the subsolution property has to be stated in terms of the lower-semicontinuous envelope $\underline{\mathcal{H}}_{*}^{\phi}$ of $\underline{\mathcal{H}}^{\phi}$, we can not a-priori state the boundary condition in terms of $F_{*}^{\phi}$. This will be possible only under additional assumptions on the coefficients; see Proposition 3.1 below.

In order to alleviate notations, we shall simply write below $F^{\phi *} \varphi(t, z)$ for $F^{\phi *}\left(t, z, D \varphi(t, z), D^{2} \varphi(t, z)\right)$, and similarly for $F_{*}^{\phi} \varphi(t, z), H_{*} \varphi(t, z), H^{*} \varphi(t, z), \overline{\mathcal{H}}^{\phi} \varphi(t, z), \underline{\mathcal{H}}^{\phi} \varphi(t, z)$ and $\underline{\mathcal{H}}_{*}^{\phi} \varphi(t, z)$. 
Finally, since the value function $V$ may not be smooth, we can only provide a PDE characterization in the discontinuous viscosity sense. We therefore introduce its semicontinuous envelopes: $\forall(t, z) \in$ $\operatorname{cl}(D)$,

$$
V_{*}(t, z):=\liminf _{\left(t^{\prime}, z^{\prime}\right) \in \operatorname{int}_{p}(D) \rightarrow(t, z)} V\left(t^{\prime}, z^{\prime}\right) \quad, \quad V^{*}(t, z):=\limsup _{\left(t^{\prime}, z^{\prime}\right) \in \operatorname{int}_{p}(D) \rightarrow(t, z)} V\left(t^{\prime}, z^{\prime}\right)
$$

\subsection{Main results}

We shall appeal to the following weak version of continuity assumption on $\mathcal{N}_{0}$ made in [5] (it will be strengthened when discussing the subsolution property on the boundary, see (ii) of Assumption 3.3 below).

Assumption 3.1. (Continuity of $\left.\mathcal{N}_{0}(x, y, q)\right)$ Let $\psi$ be Lipschitz continuous function on $[0, T] \times$ $\mathbb{R}^{d}$ and let $\mathcal{O}$ be some open subset of $[0, T] \times \mathbb{R}^{d+1}$ such that $\mathcal{N}_{0}(x, y, \psi(t, x)) \neq \emptyset$ for all $(t, x, y) \in$ $\mathcal{O}$. Then, for every $\varepsilon>0,\left(t_{0}, x_{0}, y_{0}\right) \in \mathcal{O}$ and $u_{0} \in \mathcal{N}_{0}\left(x_{0}, y_{0}, \psi\left(t_{0}, x_{0}\right)\right)$, there exists an open neighborhood $\mathcal{O}^{\prime}$ of $\left(t_{0}, x_{0}, y_{0}\right)$ and a locally Lipschitz continuous map $\hat{u}$ defined on $\mathcal{O}^{\prime}$ such that $\left|\hat{u}\left(t_{0}, x_{0}, y_{0}\right)-u_{0}\right| \leq \varepsilon$ and $\hat{u}(t, x, y) \in \mathcal{N}_{0}(x, y, \psi(t, x))$ on $\mathcal{O}^{\prime}$.

Remark 3.2. As we shall see in the statement of the following theorem (and as it will be clear from its proof), Assumption 3.1 is only required to get the supersolution property on $\partial_{Z} D$.

We can now state the main result of this section.

Theorem 3.1 (PDE characterization of the value function). The following holds:

1. If Assumption 3.1 holds, then the function $V_{*}$ is a viscosity supersolution on $\operatorname{cl}(D)$ of

$$
\begin{aligned}
& \left(-\partial_{t} \varphi+H^{*} \varphi\right)(t, x, y) \geq 0 \quad \text { if } \quad(t, x, y) \in \operatorname{int}_{p}(D) \\
& \left.\forall \phi \in \mathcal{W}^{*}(t, x),\left(-\partial_{t} \varphi+F^{\phi *} \varphi\right)(t, x, y) \geq 0 \quad \text { if } \quad(t, x, y) \in \partial_{Z} D \quad\right\} \\
& \left.\begin{array}{rl}
\varphi(T, x, y) \geq f_{*}(x, y) \quad \text { if } \quad & (t, x, y) \in \partial_{T} D, \\
& y>w(T, x), H^{*} \varphi(T, x, y)<\infty .
\end{array}\right\}
\end{aligned}
$$

2. The function $V^{*}$ is a viscosity subsolution on $\operatorname{cl}(D)$ of

$$
\begin{aligned}
&\left(-\partial_{t} \varphi+H_{*} \varphi\right)(t, x, y) \leq 0 \text { if } \quad(t, x, y) \in \operatorname{int}_{p}(D) \cup \partial_{Z} D \\
& \varphi(T, x, y) \leq f^{*}(x, y) \quad \text { if } \quad(t, x, y) \in \partial_{T} D \quad \text { and } H_{*} \varphi(T, x, y)>-\infty .
\end{aligned}
$$

The proof will be provided in Section 6 below.

Remark 3.3. Note that the above theorem does not provide the boundary condition for $V_{*}$ on the corner $\{(T, x, y): y=w(T, x)\}$. However, particular cases can be studied in more details. For instance, if $U$ is bounded, one easily checks, by using the Lipschitz continuity of $\mu_{Z}$ and $\sigma_{Z}$, that $Z_{t_{n}, z_{n}}^{\nu_{n}}(T) \rightarrow z_{0}$ in $L^{4}(\Omega)$ for any sequence $\left(t_{n}, z_{n}, \nu_{n}\right)_{n} \in D \times \mathcal{U}$ such that $\nu_{n} \in \mathcal{U}_{t_{n}, z_{n}}$ for all $n$ and $\left(t_{n}, z_{n}\right) \rightarrow\left(T, z_{0}\right)$. Since, by definition, $V\left(t_{n}, z_{n}\right) \geq \mathbb{E}\left[f\left(Z_{t_{n}, z_{n}}^{\nu_{n}}(T)\right)\right]$, the quadratic growth assumption on $f$ implies $\liminf _{n \rightarrow \infty} V\left(t_{n}, z_{n}\right) \geq f_{*}\left(z_{0}\right)$. A more general result will be stated in Proposition 3.2 when $w$ is smooth. 
To conclude this section, we now provide an equivalence result between subsolutions of $-\partial_{t} \varphi+$ $H_{*} \varphi=0$ and subsolutions of $-\partial_{t} \varphi+F_{*}^{\phi} \varphi=0$ for any $\phi \in \mathcal{W}_{*}$, under the following additional assumption:

Assumption 3.2. One of the following holds:

- Either $U$ is bounded

- or $\left|\sigma_{Y}(x, y, u)\right| \rightarrow+\infty$ as $|u| \rightarrow+\infty$ and

$$
\limsup _{|u| \rightarrow \infty, u \in U} \frac{\left|\mu_{Y}(x, y, u)\right|+\left|\mu_{X}(x, u)\right|+\left|\sigma_{X}(x, u)\right|^{2}}{\left|\sigma_{Y}(x, y, u)\right|^{2}}=0 \quad \text { for all }(x, y) \in \mathbb{R}^{d+1}
$$

and the convergence is local uniform with respect to $(x, y)$.

Remark 3.4. Note that the above assumption implies that $N_{\delta}(x, y, q)$ is compact for all $(x, y, q) \in$ $\mathbb{R}^{d+1} \times \mathbb{R}^{d}$.

Remark 3.5. This condition will be satisfied in the example of application of Section 4.

Proposition 3.1. Let Assumption 3.2 hold. Let $v$ be a upper-semicontinuous function. Assume that for all smooth function $\varphi$ and $(t, x, y) \in \partial_{Z} D$ such that $(t, x, y)$ is a strict maximum point on $\operatorname{cl}(D)$ of $v-\varphi$, we have

$$
-\partial_{t} \varphi(t, x, y)+H_{*} \varphi(t, x, y) \leq 0 .
$$

Then, for all smooth function $\varphi$ and $(t, x, y) \in \partial_{Z} D$ such that $(t, x, y)$ is a strict maximum point on $\operatorname{cl}(D)$ of $v-\varphi$ and all $\phi \in \mathcal{W}_{*}(t, x)$, we have

$$
-\partial_{t} \varphi(t, x, y)+F_{*}^{\phi} \varphi(t, x, y) \leq 0 .
$$

The proof will be provided in Section 6.3 below.

This allows to rewrite the spacial boundary condition for $V^{*}$ in terms of the more natural constrained operator $F_{*}^{\phi}$ instead of $H_{*}$.

Corollary 3.1 (Boundary conditions in the classical sense). Let Assumption 3.2 hold. Then, $V^{*}$ is a viscosity subsolution on $\operatorname{cl}(D)$ of

$$
\begin{aligned}
\left(-\partial_{t} \varphi+H_{*} \varphi\right)(t, x, y) & \leq 0 \text { if }(t, x, y) \in \operatorname{int}_{p}(D) \\
\forall \phi \in \mathcal{W}_{*}(t, x), \quad\left(-\partial_{t} \varphi+F_{*}^{\phi} \varphi\right)(t, x, y) & \leq 0 \text { if }(t, x, y) \in \partial_{Z} D .
\end{aligned}
$$

\subsection{Dirichlet boundary condition when $w$ is smooth}

Recall that the boundary $\partial_{Z} D$ corresponds to the point $(t, x, y)$ such that $y=w(t, x)$. It is therefore tempting to rewrite the boundary condition on $\partial_{Z} D$ for $V$ as $V(t, x, y)=\mathcal{V}(t, x):=V(t, x, w(t, x))$, and try to obtain a PDE characterization for $\mathcal{V}$ by performing a simple change of variables.

In this section, we shall assume that $w$ is smooth and that $\mathcal{N}_{0}(z, p)$ admits a unique argument which is continuous in $(z, p)$. This assumption is made precise as follows: 
Assumption 3.3. (i) The map $w$ is $C^{1,2}\left([0, T) \times \mathbb{R}^{d}\right)$.

(ii) There exists a locally Lipschitz map $\check{u}: \mathbb{R}^{d+1} \times \mathbb{R}^{d} \rightarrow \mathbb{R}$ such that

$$
\mathcal{N}_{0}(z, p) \neq \emptyset \Rightarrow \mathcal{N}_{0}(z, p)=\{\check{u}(z, p)\}
$$

Remark 3.6. Note that Assumption 3.1 is a consequence of Assumption 3.3

Under the above assumption, it follows from [5] and [22] that $w$ is a strong solution of (3.13) below.

Theorem 3.2 ([5] and [22]). Let Assumption 3.3 hold. Then, $\mathcal{N}_{0}(\cdot, w, D w) \neq \emptyset$ on $[0, T) \times \mathbb{R}^{d}$ and $w$ satisfies

$$
0=\min \left\{\mu_{Y}(\cdot, w, \hat{u})-\mathcal{L}_{X}^{\hat{u}} w, \mathbf{1}_{\hat{u} \in \operatorname{int}(U)}\right\} \quad \text { on }[0, T) \times \mathbb{R}^{d}
$$

where

$$
\hat{u}:=\check{u}(\cdot, w, D w) .
$$

Moreover, for all $x \in \mathbb{R}^{d}$ and smooth function $\phi$ such that $x$ reaches a local minimum of $w(T, \cdot)-\phi$, the set $\mathcal{N}_{0}(x, w(T, x), D \phi(x))$ is non-empty.

Remark 3.7. Theorem 3.2 is proved in [5] and [22] when the set of controls is $\mathcal{U}$ in place of $\mathcal{U}^{t}$, as a consequence of the geometric dynamic programming principle of Soner and Touzi [21]. Even if the formulation is slightly different here, the proofs of the above papers can be entirely reproduced for $\mathcal{U}^{t}$ in place of $\mathcal{U}$ by appealing to the version of geometric dynamic programming principle stated in Theorem 2.1 and Corollary 2.1 above.

Also observe that the first assertion in Assumption 3.3 combined with the discussion of Section 3.1 (see (3.3) and (3.6)) implies that the boundary condition on $\partial_{Z} D$ can be simplified and written in terms of $F_{\delta, \gamma}^{w}$. Using this simplification and computing the derivatives of $\mathcal{V}$ in terms of the derivatives of $V$ and $w$, we deduce from Theorem 3.1, Corollary 3.1 and Theorem 3.2 that, at least at a formal level, $\mathcal{V}$ is a solution of (3.14)-(3.15) below.

To be more precise, the viscosity solution property will be stated in terms of

$$
\mathcal{V}_{*}(t, x):=V_{*}(t, x, w(t, x)) \quad \text { and } \quad \mathcal{V}^{*}(t, x):=V^{*}(t, x, w(t, x)),
$$

for $(t, x) \in[0, T] \times \mathbb{R}^{d}$. Since $w$ is continuous, $\mathcal{V}_{*}\left(\right.$ resp. $\left.\mathcal{V}^{*}\right)$ is lower-semicontinuous (resp. uppersemicontinuous).

Our proof of the subsolution property will also require the following additional technical assumption:

Assumption 3.4. The condition (3.11) holds. Moreover, for all sequence $\left(t_{n}, x_{n}\right)_{n}$ in $[0, T) \times \mathbb{R}^{d}$ such that $\left(t_{n}, x_{n}\right) \rightarrow\left(t_{0}, x_{0}\right) \in[0, T) \times \mathbb{R}^{d}$ such that $\hat{u}\left(t_{0}, x_{0}\right) \in \operatorname{int}(U)$, we have $\liminf _{n \rightarrow \infty} \inf _{u \in U}\left[n\left(\mathcal{L}_{X}^{u} w\left(t_{n}, x_{n}\right)-\mu_{Y}\left(x_{n}, w\left(t_{n}, x_{n}\right), u\right)\right)+n^{2}\left|N^{u}\left(x_{n}, w\left(t_{n}, x_{n}\right), D w\left(t_{n}, x_{n}\right)\right)\right|^{2}\right]>-\infty$. 
We can now state the main result of this section.

Proposition 3.2. Let Assumption 3.3 hold, and assume that $V$ and $w$ have polynomial growth. Then, $\mathcal{V}_{*}$ is a viscosity supersolution on $[0, T] \times \mathbb{R}^{d}$ of

$$
\begin{cases}\left(-\mathcal{L}_{X}^{\hat{u}} \varphi\right) \mathbf{1}_{\hat{u} \in \operatorname{int}(U)}=0 \quad \text { on }[0, T) \times \mathbb{R}^{d} \\ \varphi(T, \cdot) \geq f_{*}(\cdot, w(T, \cdot)) \quad \text { if } \limsup _{\substack{\left(t^{\prime}, x^{\prime}\right) \rightarrow(T, \cdot) \\ t<T}}\left|\hat{u}\left(t^{\prime}, x^{\prime}\right)\right|<\infty \quad \text { and } \mathcal{W}^{*}(T, \cdot) \neq \emptyset .\end{cases}
$$

Moreover, if in addition Assumption 3.4 holds, then $\mathcal{V}^{*}$ is a viscosity subsolution on $[0, T] \times \mathbb{R}^{d}$ of

$$
\left\{\begin{array}{l}
\left(-\mathcal{L}_{X}^{\hat{u}} \varphi\right) \mathbf{1}_{\hat{u} \in \operatorname{int}(U)}=0 \quad \text { in }[0, T) \times \mathbb{R}^{d}, \\
\varphi(T, \cdot) \leq f^{*}(\cdot, w(T, \cdot)) \quad \text { if } \mathcal{W}_{*}(T, \cdot) \neq \emptyset .
\end{array}\right.
$$

See Section 6.4 for the proof.

Remark 3.8. (i) Note that $\mathbf{1}_{\hat{u} \in \operatorname{int}(U)}=1$ if $U=\mathbb{R}^{d}$. Even if $U \neq \mathbb{R}^{d}$, the PDE simplifies whenever $w$ solves

$$
\mu_{Y}(\cdot, w, \hat{u})-\mathcal{L}_{X}^{\hat{u}} w=0 \quad \text { on }[0, T) \times \mathbb{R}^{d}
$$

It will be clear from the proof of Proposition 3.2 that in this case $\mathcal{V}_{*}$ is a viscosity supersolution on $[0, T) \times \mathbb{R}^{d}$ of $-\mathcal{L}_{X}^{\hat{u}} \varphi=0$. Similarly, $\mathcal{V}^{*}$ is a viscosity subsolution of the same equation if Assumption 3.4 holds without the condition $\hat{u}\left(t_{0}, x_{0}\right) \in \operatorname{int}(U)$. We refer to [8] for situations in Mathematical Finance where $w$ is actually a viscosity solution of the above equation, although $U \neq \mathbb{R}^{d}$.

(ii) The Assumption 3.4 will be satisfied in our example of application of Section 4. Note that it also holds in our general framework when $\mu_{Z}$ and $\left|\sigma_{X}\right|^{2}$ are locally Lipschitz in u, locally uniformly in the other variables, and

$$
\left|N^{u}(x, y, q)-N^{u^{\prime}}(x, y, q)\right|^{2} \geq \rho_{1}\left(x, y, q, u, u^{\prime}\right)\left|u-u^{\prime}\right|^{2}-\rho_{2}\left(x, y, q, u, u^{\prime}\right)
$$

for some locally bounded maps $\rho_{1}, \rho_{2}$ such that $\rho_{1}>0$ locally uniformly. Indeed, in this case, we have $\hat{u}\left(t_{n}, x_{n}\right) \rightarrow \hat{u}\left(t_{0}, x_{0}\right) \in \operatorname{int}(U)$ and Theorem 3.2 implies that

$$
\begin{aligned}
& n\left(\mathcal{L}_{X}^{u} w\left(t_{n}, x_{n}\right)-\mu_{Y}\left(x_{n}, w\left(t_{n}, x_{n}\right), u\right)\right)+n^{2}\left|N^{u}\left(x_{n}, w\left(t_{n}, x_{n}\right), D w\left(t_{n}, x_{n}\right)\right)\right|^{2} \\
& =n\left(\mathcal{L}_{X}^{u} w\left(t_{n}, x_{n}\right)-\mu_{Y}\left(x_{n}, w\left(t_{n}, x_{n}\right), u\right)\right)-n\left(\mathcal{L}_{X}^{\hat{u}\left(t_{n}, x_{n}\right)} w\left(t_{n}, x_{n}\right)-\mu_{Y}\left(x_{n}, w\left(t_{n}, x_{n}\right), \hat{u}\left(t_{n}, x_{n}\right)\right)\right) \\
& +n^{2}\left|N^{u}\left(x_{n}, w\left(t_{n}, x_{n}\right), D w\left(t_{n}, x_{n}\right)\right)-N^{\hat{u}\left(t_{n}, x_{n}\right)}\left(x_{n}, w\left(t_{n}, x_{n}\right), D w\left(t_{n}, x_{n}\right)\right)\right|^{2} \\
& \geq-n C_{1}\left|u-\hat{u}\left(t_{n}, x_{n}\right)\right|+n^{2} C_{2}\left|u-\hat{u}\left(t_{n}, x_{n}\right)\right|^{2}-C_{3} \geq-\frac{C_{1}^{2}}{4 C_{2}}-C_{3}
\end{aligned}
$$

for some constant $C_{1}, C_{2}, C_{3}>0$. In view of (i), it also holds without the condition $\hat{u}\left(t_{0}, x_{0}\right) \in$ $\operatorname{int}(U)$ when $w$ solves (3.16).

(iii) If the map $\hat{u}$ is locally bounded, then the condition $\lim \sup \left\{\left|\hat{u}\left(t^{\prime}, x^{\prime}\right)\right|,\left(t^{\prime}, x^{\prime}\right) \rightarrow(T, \cdot), t^{\prime}<\right.$ $T\}<\infty$ holds when $x \mapsto \lim \sup \left\{\left|D w\left(t^{\prime}, x^{\prime}\right)\right|,\left(t^{\prime}, x^{\prime}\right) \rightarrow(T, x), t^{\prime}<T\right\}$ is locally bounded. 
Under the above assumptions, we can then replace the boundary condition of Theorem 3.1 and Corollary 3.1 by a simple Dirichlet condition $V(t, x, y)=\mathcal{V}(t, x)$ on $\partial_{Z} D$, where $\mathcal{V}$ can be computed by solving $-\mathcal{L}_{X}^{\hat{u}} \varphi=0$ on $[0, T) \times \mathbb{R}^{d}$ with the corresponding terminal condition at $t=T$.

Obviously, the construction of the associated numerical schemes requires at least comparison results for the corresponding PDEs which are a-priori highly non-linear and not continuous. We leave this important point for further research.

\section{Example of application in mathematical finance}

In order to illustrate our results, we detail in this section an application to the problem of terminal wealth maximization under a super-replication constraint.

\subsection{The optimization problem}

Namely, we consider a financial market consisting of a riskless asset, whose price process is normalized to 1 for simplicity, and one risky asset whose dynamics is given by

$$
X_{t, x}(s)=x+\int_{t}^{s} X_{t, x}(u) \mu\left(X_{t, x}(u)\right) d u+\int_{t}^{s} X_{t, x}(u) \sigma\left(X_{t, x}(u)\right) d W_{u}
$$

where $x \in[0, \infty) \mapsto(x \mu(x), x \sigma(x))$ is Lipschitz continuous and

$$
\sigma \geq \varepsilon \text { and }|\mu / \sigma| \leq \varepsilon^{-1} \text { for some real constant } \varepsilon>0 .
$$

The control process $\nu$ corresponding to the amount of money invested in the risky asset is valued in $U=\mathbb{R}$. Under the self-financing condition, the value of the corresponding portfolio $Y^{\nu}$ is given by

$$
Y_{t, x, y}^{\nu}(s)=y+\int_{t}^{s} \nu_{u} \frac{d X_{t, x}(u)}{X_{t, x}(u)}=y+\int_{t}^{s} \nu_{u} \mu\left(X_{t, x}(u)\right) d u+\int_{t}^{s} \nu_{u} \sigma\left(X_{t, x}(u)\right) d W_{u} .
$$

We consider a fund manager whose preferences are given by a $C^{2}$ increasing strictly concave utility function $U$ on $\mathbb{R}_{+}$satisfying the classical Inada conditions:

$$
U^{\prime}(0+)=+\infty \quad \text { and } \quad U^{\prime}(+\infty)=0 .
$$

We assume that his objective is to maximize his expected utility of wealth under the constraint that it performs better than the benchmark (or super-hedge some European option's payoff) $\psi\left(X_{t, x}(T)\right.$ ), with $\psi$ a continuous function with polynomial growth.

The corresponding optimal control problem under stochastic constraint is given by:

$$
V(t, x, y):=\sup _{\nu \in \mathcal{U}_{t, x, y}} \mathbb{E}\left[U\left(Y \nu_{t, x, y}(T)\right)\right]
$$

with

$$
\mathcal{U}_{t, x, y}:=\left\{\nu \in \mathcal{U}^{t}, Y_{t, x, y}^{\nu}(T) \geq \psi\left(X_{t, x}(T)\right) \quad \mathbb{P}-\text { a.s. }\right\}
$$




\subsection{The PDE characterization}

Note that under the condition (4.1), we simply have

$$
\mathcal{N}_{\delta}(x, y, q)=\{u \in \mathbb{R}:|u \sigma(x)-x \sigma(x) q| \leq \delta\}=\{x q+\delta \zeta / \sigma(x),|\zeta| \leq 1\}
$$

Moreover, the function $w$ is given by

$$
w(t, x)=\mathbb{E}^{\mathbb{Q}_{t, x}}\left[\psi\left(X_{t, x}(T)\right)\right]
$$

where $\mathbb{Q}_{t, x} \sim \mathbb{P}$ is defined by

$$
\frac{d \mathbb{Q}_{t, x}}{d \mathbb{P}}=\exp \left(-\frac{1}{2} \int_{t}^{T}\left|\lambda\left(X_{t, x}(s)\right)\right|^{2} d s-\int_{t}^{T} \lambda\left(X_{t, x}(s)\right) d W_{s}\right) \quad \text { with } \lambda:=\mu / \sigma,
$$

see e.g. [15], and is a continuous viscosity solution on $[0, T) \times(0, \infty)$ of

$$
\begin{aligned}
0 & =-\partial_{t} \varphi(t, x)-\frac{1}{2} x^{2} \sigma^{2}(x) D^{2} \varphi(t, x) \\
& =\sup _{u \in \mathcal{N}_{0}(x, \varphi(t, x), D \varphi(t, x))}\left(u \mu(x)-\partial_{t} \varphi(t, x)-x \mu(x) D \varphi(t, x)-\frac{1}{2} x^{2} \sigma^{2}(x) D^{2} \varphi(t, x)\right)
\end{aligned}
$$

with the terminal condition $w(T, \cdot)=\psi$.

Also note that Assumptions 3.1 and 3.2 trivially hold in this context. It then follows that we can apply Theorem 3.1 and Corollary 3.1.

Moreover, if $\mu$ and $\sigma$ are smooth enough, it follows from standard estimates that $w \in C^{1,2}([0, T) \times$ $(0, \infty))$. If $\psi$ is also Lipschitz continuous, then $|D w|$ is locally bounded and so is $(t, x) \mapsto \hat{u}(t, x)=$ $x D w(t, x)$, recall (4.3). The conditions of Assumption 3.4 are also satisfied, see (ii) of Remark 3.8. It then follows from Proposition 3.2 that $\mathcal{V}_{*}$ and $\mathcal{V}^{*}$ are viscosity super- and subsolutions on $[0, T) \times(0, \infty)$ of

$$
0=-\partial_{t} \varphi(t, x)-x \mu(x) D \varphi(t, x)-\frac{1}{2} x^{2} \sigma^{2}(x) D^{2} \varphi(t, x),
$$

with the terminal condition, assuming that $\psi \in C^{2}$,

$$
\varphi(T, x)=U(\psi(x)) .
$$

Standard comparison results and the Feynman Kac formula thus imply that

$$
\mathcal{V}_{*}(t, x)=\mathcal{V}^{*}(t, x)=\mathbb{E}\left[U\left(\psi\left(X_{t, x}(T)\right)\right)\right]=: \mathcal{V}(t, x),
$$

which provides an "explicit" Dirichlet boundary condition for $V$ on $\{y=w(t, x)\}$.

Summing up the above results, we obtain that $V_{*}$ is a viscosity supersolution of

$$
\begin{aligned}
& -\partial_{t} \varphi(t, x, y)+H^{*} \varphi(t, x, y) \geq 0 \quad \text { if } \quad t<T, y>w(t, x) \\
& \varphi(t, x, y) \geq \mathcal{V}(t, x) \quad \text { if } \quad t<T, y=w(t, x) \\
& \varphi(T, x, y) \geq U(y) \quad \text { if } \quad y \geq \psi(x) \text {, }
\end{aligned}
$$


and that $V^{*}$ is a viscosity subsolution of

$$
\begin{aligned}
-\partial_{t} \varphi(t, x, y)+H_{*} \varphi(t, x, y) \leq 0 & \text { if } \quad t<T, y>w(t, x) \\
\varphi(t, x, y) \leq \mathcal{V}(t, x) & \text { if } \quad t<T, y=w(t, x) \\
\varphi(T, x, y) \leq U(y) & \text { if } \quad y \geq \psi(x),
\end{aligned}
$$

where

$$
H(x, y, q, A):=\inf _{u \in \mathbb{R}}\left(-\mu(x)\left\langle\left(\begin{array}{l}
x \\
u
\end{array}\right), q\right\rangle-\frac{1}{2} \sigma(x)^{2} \operatorname{Tr}\left[\left[\begin{array}{cc}
x^{2} & x u \\
x u & u^{2}
\end{array}\right] A\right]\right) .
$$

\subsection{Explicit resolution}

In order to solve the above PDE explicitly, we proceed as in [5] and introduce the Fenchel-Legendre dual transform associated to $V^{*}$ with respect to the $y$ variable:

$$
\tilde{V}^{*}(t, x, \gamma):=\sup _{y \in[w(t, x), \infty)}\left\{V^{*}(t, x, y)-y \gamma\right\}, \quad(t, x, \gamma) \in[0, T] \times(0, \infty) \times \mathbb{R}
$$

where we use the usual extension $V^{*}(t, x, y)=\infty$ if $y<w(t, x)$. Note that $\tilde{V}^{*}(t, x, \gamma)=\infty$ if $\gamma<0$ since $V$ is clearly non-decreasing in $y$. The main interest in considering the dual transform of the value function is to get rid of the non linear terms in the above PDE. By the same arguments as in Section 4 in [5], we indeed obtain that $\tilde{V}^{*}$ is an upper-semicontinuous viscosity subsolution of $[0, T] \times(0, \infty) \times(0, \infty)$ of

$$
\begin{array}{r}
-\mathcal{L}_{X} \varphi+x \sigma(x) \gamma \lambda(x) \frac{\partial^{2}}{\partial x \partial \gamma} \varphi-\frac{1}{2} \gamma^{2} \lambda(x)^{2} \frac{\partial^{2}}{\partial \gamma^{2}} \varphi \leq 0 \quad \text { if } \quad t<T, \frac{\partial}{\partial \gamma} \varphi(t, x, \gamma)<-w(t, x) \\
\varphi(T, x, \gamma) \leq U(I(\gamma) \vee \psi(x))-\gamma[I(\gamma) \vee \psi(x)] \quad \text { if } \quad t=T, \frac{\partial}{\partial \gamma} \varphi(T, x, \gamma) \leq-\psi(x),
\end{array}
$$

where $I:=\left(U^{\prime}\right)^{-1}$ and where we used (4.2) to derive the boundary condition. Let us now define

$$
\bar{V}(t, x, \gamma):=\mathbb{E}\left[U\left[I\left(\Gamma_{t, x, \gamma}(T)\right) \vee \psi\left(X_{t, x}(T)\right)\right]\right]-\mathbb{E}\left[\Gamma_{t, x, \gamma}(T)\left\{I\left(\Gamma_{t, x, \gamma}(T)\right) \vee \psi\left(X_{t, x}(T)\right)\right\}\right],
$$

where $\Gamma_{t, x, \gamma}$ has the dynamics

$$
\Gamma_{t, x, \gamma}(s)=\gamma+\int_{t}^{s} \lambda\left(X_{t, x}(u)\right) \Gamma_{t, \gamma}(u) d W_{u} .
$$

One easily checks that $\bar{V}$ is a continuous viscosity solution of the above PDE satisfying $\bar{V}(T, x, \gamma)=$ $\gamma[I(\gamma) \vee \psi(x)]-U(I(\gamma) \vee \psi(x))$ and, recalling (4.4),

$$
\frac{\partial}{\partial \gamma} \bar{V}(t, x, \gamma)=-\mathbb{E}\left[\Gamma_{t, x, 1}(T)\left\{I\left(\Gamma_{t, x, \gamma}(T)\right) \vee \psi\left(X_{t, x}(T)\right)\right\}\right] \leq-\mathbb{E}^{\mathbb{Q} t, x}\left[\psi\left(X_{t, x}(T)\right)\right]=-w(t, x) .
$$

We then deduce from standard comparison results, see e.g. [11], that

$$
\tilde{V}^{*}(t, x, \gamma) \leq \bar{V}(t, x, \gamma)
$$


This leads to

$$
\begin{aligned}
V^{*}(t, x, y) & \leq \inf _{\gamma>0}\{\bar{V}(t, x, \gamma)+y \gamma\} \\
& =\inf _{\gamma>0}\left\{\mathbb{E}\left[U\left[I\left(\Gamma_{t, x, \gamma}(T)\right) \vee \psi\left(X_{t, x}(T)\right)\right]\right]-\mathbb{E}\left[\Gamma_{t, x, \gamma}(T)\left\{I\left(\Gamma_{t, x, \gamma}(T)\right) \vee \psi\left(X_{t, x}(T)\right)\right\}\right]+y \gamma\right\}
\end{aligned}
$$

Computing the optimal argument $\hat{\gamma}(t, x, y)$ in the above expression, recall (4.2), we obtain that

$$
y:=\mathbb{E}^{\mathbb{Q} t, x}\left[I\left(\Gamma_{t, x, \hat{\gamma}(t, x, y)}(T)\right) \vee \psi\left(X_{t, x}(T)\right)\right],
$$

and therefore

$$
V^{*}(t, x, y) \leq \bar{V}(t, x, \hat{\gamma}(t, x, y))+y \hat{\gamma}(t, x, y)=\mathbb{E}\left[U\left[I\left(\Gamma_{t, x, \hat{\gamma}(t, x, y)}(T)\right) \vee \psi\left(X_{t, x}(T)\right)\right]\right] .
$$

Furthermore, (4.7), the martingale representation theorem and (4.1) ensure that we can find some $\mathbb{P}$ - a.s.-square integrable predictable process $\hat{\nu}$, independent of $\mathcal{F}_{t}$ such that

$$
Y_{t, y, x}^{\hat{\nu}}(T)=I\left(\Gamma_{t, \hat{\gamma}(t, x, y)}(T)\right) \vee \psi\left(X_{t, x}(T)\right) .
$$

In particular, this implies that $Y_{t, y}^{\hat{\nu}}(T) \geq \psi\left(X_{t, x}(T)\right)$ so that $\nu \in \mathcal{U}_{t, x, y}$ if $I\left(\Gamma_{t, \hat{\gamma}(t, x, y)}(T)\right) \in$ $L^{2+\varepsilon}(\Omega, \mathbb{Q})$ for some $\varepsilon>0$, recall that $\psi$ has polynomial growth so that $\psi\left(X_{t, x}(T)\right) \in L^{p}(\Omega, \mathbb{Q})$ for any $p \geq 1$. Therefore, under the above assumption, the value function is given by

$$
\begin{aligned}
V(t, x, y) & =\mathbb{E}\left[U\left[I\left(\Gamma_{t, x, \hat{\gamma}(t, x, y)}(T)\right) \vee \psi\left(X_{t, x}(T)\right)\right]\right] \\
\text { with } y & =\mathbb{E}^{\mathbb{Q} t, x}\left[I\left(\Gamma_{t, x, \hat{\gamma}(t, x, y)}(T)\right) \vee \psi\left(X_{t, x}(T)\right)\right] .
\end{aligned}
$$

This is the result obtained in [16] via duality methods in the case of a European guarantee.

Remark 4.1. The condition $I\left(\Gamma_{t, x, \hat{\gamma}(t, x, y)}(T)\right) \in L^{2+\varepsilon}(\Omega, \mathbb{Q})$ is satisfied for many examples of applications. This is in particular the case for $U(r):=r^{p}$ for $0<p<1$. In the case where it does not hold, one needs to relax the set of controls by allowing $\nu$ to be only $\mathbb{P}-$ a.s.-square integrable. The proofs of our general results can be easily adapted to this context in this example.

Remark 4.2. We have assumed that $\psi \in C^{2}$. If this is not the case, we can always consider sequences of smooth functions $\left(\bar{\psi}_{n}\right)_{n}$ and $\left(\underline{\psi}_{n}\right)_{n}$ such that $\underline{\psi}_{n} \leq \psi \leq \bar{\psi}_{n}$ and $\underline{\psi}_{n}, \bar{\psi}_{n} \rightarrow \psi$ pointwise. Letting $\underline{V}_{n}, \bar{V}_{n}$ be the value functions associated to $\underline{\psi}_{n}, \bar{\psi}_{n}$ instead of $\psi$, we clearly have $\underline{V}_{n} \leq V \leq$ $\bar{V}_{n}$. Letting $n \rightarrow \infty$ and using (4.8) applied to $\underline{V}_{n}, \overline{\bar{V}}_{n}$ shows that (4.8) holds for $V$ too.

\section{$5 \quad$ Possible extensions}

\subsection{Constraints in moment or probability}

In this section, we explain how the results of the Section 3 can be adapted to stochastic control problems under moment constraints of the following form

$$
\tilde{V}(t, z ; p):=\sup _{\nu \in \tilde{\mathcal{U}}_{t, z, p}} \mathbb{E}\left[f\left(Z_{t, z}^{\nu}(T)\right)\right]
$$


where

$$
\tilde{\mathcal{U}}_{t, z, p}:=\left\{\nu \in \mathcal{U}^{t}: \mathbb{E}\left[g\left(Z_{t, z}^{\nu}(T)\right)\right] \geq p\right\} \quad, p \in \mathbb{R}
$$

The main idea consists in adding a new controlled process $P_{t, p}^{\alpha}$ as done in Bouchard, Touzi and Elie [5] for stochastic control problem with controlled loss.

Namely, let $\mathcal{A}$ denote the set of $\mathbb{F}$-progressively measurable $\mathbb{R}^{d}$-valued square integrable processes and denote by $\mathcal{A}^{t}$ the subset of elements of $\mathcal{A}$ which are independent of $\mathcal{F}_{t}$. To $\alpha \in \mathcal{A}^{t}$ and initial conditions $(t, p) \in[0, T] \times \mathbb{R}$, we associate the controlled process $P_{t, p}^{\alpha}$ defined for $t \leq s \leq T$ by

$$
P_{t, p}^{\alpha}(s)=p+\int_{t}^{s} \alpha_{r}^{\top} d W_{r}
$$

and introduce the set $\overline{\mathcal{U}}_{t, z, p}$ of controls of the form $(\nu, \alpha) \in \mathcal{U}^{t} \times \mathcal{A}^{t}$ such that

$$
\bar{g}\left(Z_{t, z}^{\nu}(T), P_{t, p}^{\alpha}(T)\right) \geq 0 \mathbb{P}-\text { a.s. }
$$

where $\bar{g}(Z, P):=g(Z)-P$.

Lemma 5.1. Fix $(t, z, p) \in[0, T] \times \mathbb{R}^{d+1} \times \mathbb{R}$ and $\nu \in \mathcal{U}^{t}$ such that $g\left(Z_{t, z}^{\nu}(T)\right) \in L^{2}(\Omega)$. Then, $\nu \in \tilde{\mathcal{U}}_{t, z, p}$ if and only if there exists $\alpha \in \mathcal{A}^{t}$ such that $(\nu, \alpha) \in \overline{\mathcal{U}}_{t, z, p}$.

Proof. Fix $\nu \in \tilde{\mathcal{U}}_{t, z, p}$. Since $g\left(Z_{t, z}^{\nu}(T)\right) \in L^{2}(\Omega)$ and is independent of $\mathcal{F}_{t}$, we can find $\alpha \in \mathcal{A}^{t}$ such that $g\left(Z_{t, z}^{\nu}(T)\right)=\mathbb{E}\left[g\left(Z_{t, z}^{\nu}(T)\right)\right]-p+P_{t, p}^{\alpha}(T)$. Since $\mathbb{E}\left[g\left(Z_{t, z}^{\nu}(T)\right)\right] \geq p$, it follows that $\bar{g}\left(Z_{t, Z}^{\nu}(T), P_{t, p}^{\alpha}(T)\right) \geq 0$. The converse assertion follows from the martingale property of $P_{t, p}^{\alpha}$, for all $(\nu, \alpha) \in \overline{\mathcal{U}}_{t, z, p}$.

This readily implies that $\tilde{V}=\bar{V}$ where

$$
\bar{V}(t, z, p):=\sup _{\nu \in \overline{\mathcal{U}}_{t, z, p}} \mathbb{E}\left[f\left(Z_{t, z}^{\nu}(T)\right)\right]
$$

Proposition 5.1. Fix $(t, z, p) \in[0, T] \times \mathbb{R}^{d+1} \times \mathbb{R}$, and assume that $g\left(Z_{t, z}^{\nu}(T)\right) \in L^{2}(\Omega)$ for all $\nu \in \mathcal{U}^{t}$. Then, $\tilde{V}(t, z ; p)=\bar{V}(t, z, p)$.

Remark 5.1. (i) If $g$ has linear growth then the condition $g\left(Z_{t, z}^{\nu}(T)\right) \in L^{2}(\Omega)$ is satisfied since the diffusion has Lipschitz continuous coefficients and, by definition of $\mathcal{U}, \nu$ is square integrable.

(ii) Note that, if $U$ is bounded, then the condition (3.11) holds for the augmented system $\left(Z_{t, z}^{\nu}, P_{t, p}^{\alpha}\right)$ when looking at $P_{t, p}^{\alpha}$ as a new $Y$ component and the former $Z_{t, z}^{\nu}$ as a new $X$ component:

$$
\limsup _{|(u, \alpha)| \rightarrow \infty,(u, \alpha) \in U \times \mathbb{R}}\left\{\frac{\left|\mu_{Z}(\cdot, u)\right|+\left|\sigma_{Z}(\cdot, u)\right|^{2}}{|\alpha|^{2}}\right\}=\limsup _{|\alpha| \rightarrow \infty} \sup _{u \in U}\left\{\frac{\left|\mu_{Z}(\cdot, u)\right|+\left|\sigma_{Z}(\cdot, u)\right|^{2}}{|\alpha|^{2}}\right\}=0 \quad,
$$

on $\mathbb{R}^{d+1}$ locally uniformly.

(iii) Under the integrability condition of Proposition 5.1, the PDE characterization of Section 3 can thus be applied to the value function $\bar{V}=\tilde{V}$. 
One can similarly apply the results of the preceding section to problems with constraint in probability of the form

$$
\check{V}(t, z ; p):=\sup _{\nu \in \check{\mathcal{U}}_{t, z, p}} \mathbb{E}\left[f\left(Z_{t, z}^{\nu}(T)\right)\right] \quad \text { with } \quad \check{\mathcal{U}}_{t, z, p}:=\left\{\nu \in \mathcal{U}^{t}: \mathbb{P}\left[g\left(Z_{t, z}^{\nu}(T)\right) \geq 0\right] \geq p\right\}
$$

by observing that

$$
\mathbb{P}\left[g\left(Z_{t, z}^{\nu}(T)\right) \geq 0\right]=\mathbb{E}\left[\mathbf{1}_{\left\{g\left(Z_{t, z}^{\nu}(T)\right) \geq 0\right\}}\right]
$$

In this case, we obtain

$$
\check{V}(t, z ; p)=\sup \left\{\mathbb{E}\left[f\left(Z_{t, z}^{\nu}(T)\right)\right],(\nu, \alpha) \in \mathcal{U}^{t} \times \mathcal{A}^{t} \text { s.t. } \mathbf{1}_{\left\{g\left(Z_{t, z}^{\nu}(T)\right) \geq 0\right\}}-P_{t, p}^{\alpha}(T) \geq 0 \mathbb{P}-\text { a.s. }\right\} .
$$

Remark 5.2. Following [5], we could restrict ourselves to controls $\alpha$ such that $P_{t, p}^{\alpha}$ evolves in $[0,1]$, which is the natural domain for the $p$ variable. Note that, in any case, one should also discuss the boundary conditions at $p=0$ and $p=1$, as in [5]. We leave this non-trivial point to further research.

\subsection{Almost sure constraints on $[0, T]$}

An important extension consists in considering a.s. constraints on the whole time interval $[0, T]$ :

$$
V^{\mathrm{a}}(t, z):=\sup _{\nu \in \mathcal{U}_{t, z}^{\mathrm{a}}} \mathbb{E}\left[f\left(Z_{t, z}^{\nu}(T)\right)\right]
$$

where

$$
\mathcal{U}_{t, z}^{\mathrm{a}}:=\left\{\nu \in \mathcal{U}^{t}: g\left(Z_{t, z}^{\nu}(s)\right) \geq 0 \forall s \in[t, T] \mathbb{P}-\text { a.s. }\right\}
$$

This corresponds to a classical state constraint problem on the spacial domain

$$
\mathcal{O}:=\left\{z \in \mathbb{R}^{d+1}: g(z) \geq 0\right\} .
$$

This case can be treated exactly as the one considered in the present paper, after replacing $D$ and $w$ by $D^{\mathrm{a}}$ and $w^{\mathrm{a}}$ defined by

$$
\begin{aligned}
& D^{\mathrm{a}}:=\left\{(t, z) \in[0, T] \times \mathbb{R}^{d+1}: g\left(Z_{t, z}^{\nu}(s)\right) \geq 0 \forall s \in[t, T] \mathbb{P}-\text { a.s. for some } \nu \in \mathcal{U}^{t}\right\} \\
& w^{\mathrm{a}}(t, x):=\inf \left\{y \in \mathbb{R}:(t, x, y) \in D^{\mathrm{a}}\right\} .
\end{aligned}
$$

This is due to the fact that the geometric dynamic programming principle of Theorem 2.1 and Corollary 2.1 can be extended to $D^{\mathrm{a}}$ and $w^{\mathrm{a}}$, see Bouchard and Nam [6]. Repeating the arguments of Section 6 then allows to extend to $V^{\text {a }}$ the results of Section 3.2 and Section 3.3.

We should note that the PDE characterization of $w^{\text {a }}$ has been obtained in Bouchard and Nam [6] only for a particular situation which arises in finance. However, it would not be difficult to derive the PDE associated to $w^{\mathrm{a}}$ in our more general framework by combining the arguments of [5] and [6]. We would obtain the same characterization as in [5] but only on the domain $g_{*}\left(x, w^{\mathrm{a} *}(t, x)\right)>0$ 
for the subsolution property, and with the additional inequality $g^{*}\left(x, w_{*}^{\mathrm{a}}(t, x)\right) \geq 0$ on the whole domain, i.e. $w^{\mathrm{a}}$ would be a discontinuous viscosity solution of

$$
\begin{aligned}
\min \left\{\sup _{u \in \mathcal{N}_{0}\left(\cdot, w^{\mathrm{a}}, D w^{\mathrm{a}}\right)}\left(\mu_{Y}\left(\cdot, w^{\mathrm{a}}, u\right)-\mathcal{L}_{X}^{u} w^{\mathrm{a}}\right), g\left(\cdot, w^{\mathrm{a}}\right)\right\} & =0 \text { in }[0, T) \times \mathbb{R}^{d} \\
g\left(\cdot, w^{\mathrm{a}}(T, \cdot)\right) & =0 \text { in } \mathbb{R}^{d} .
\end{aligned}
$$

As already argued in the introduction, it is important to notice that our approach would not require to impose strong conditions on $\mathcal{O}$ and the coefficients in order to ensure that $Z_{t, z}^{\nu}$ can actually be reflected on the boundary of $\mathcal{O}$. The only important assumption is that $D^{\mathrm{a}}$ is non empty. By definition of $D^{\text {a }}$ and the geometric dynamic programming principle, this condition will be automatically satisfied on the boundary of $D^{\mathrm{a}} \subset \mathcal{O}$.

We think that this approach is more natural for state constraint problems and we hope that it will open the door to new results in this field. A precise study is left for further research.

\section{Proof of the PDE characterizations}

\subsection{Dynamic programming principle}

As usual, we first need to provide a dynamic programming principle for our control problem (2.1).

Theorem 6.1 (Dynamic Programming Principle). Fix $(t, z) \in \operatorname{int}_{p}(D)$ and let $\left\{\theta^{\nu}, \nu \in \mathcal{U}\right\}$ be a family of stopping times with values in $[t, T]$. Then,

$$
\begin{aligned}
V(t, z) & \leq \sup _{\nu \in \mathcal{U}_{t, z}} \mathbb{E}\left[f^{*}\left(Z_{t, z}^{\nu}\left(\theta^{\nu}\right)\right) \mathbf{1}_{\theta^{\nu}=T}+V^{*}\left(\theta^{\nu}, Z_{t, z}^{\nu}\left(\theta^{\nu}\right)\right) \mathbf{1}_{\theta^{\nu}<T}\right], \\
V(t, z) & \geq \sup _{\nu \in \mathcal{U}_{t, z}} \mathbb{E}\left[f_{*}\left(Z_{t, z}^{\nu}\left(\theta^{\nu}\right)\right) \mathbf{1}_{\theta^{\nu}=T}+V_{*}\left(\theta^{\nu}, Z_{t, z}^{\nu}\left(\theta^{\nu}\right)\right) \mathbf{1}_{\theta^{\nu}<T}\right]
\end{aligned}
$$

where $V_{*}\left(\right.$ resp. $\left.V^{*}\right)$ denotes the lower-semicontinuous (resp. upper-semicontinuous) envelope of $V$.

Proof. In this proof we denote by $\omega^{r}:=\left(\omega_{t \wedge r}\right)_{t \leq T}$ the stopped canonical path, and by $\mathbf{T}_{r}(\omega):=$ $\left(\omega_{s+r}-\omega_{r}\right)_{s \leq T-r}$ the shifted canonical path, $r \in[0, T]$. For ease of notations, we omit the dependence of $\theta^{\nu}$ with $\nu$ and simply write $\theta$.

1. We start with the first inequality. To see that it holds, note that, by the flow property, for any $\nu \in \mathcal{U}_{t, z}:$

$$
\begin{aligned}
\mathbb{E}\left[f\left(Z_{t, z}^{\nu}(T)\right)\right] & =\iint f\left(Z_{\theta(\omega), Z_{t, z}^{\nu}(\theta)(\omega)}^{\nu(\omega)}(T)\left(\omega^{\theta(\omega)}+\mathbf{T}_{\theta(\omega)}(\tilde{\omega})\right)\right) d \mathbb{P}(\omega) d \mathbb{P}(\tilde{\omega}) \\
& =\int \mathbb{E}\left[J\left(\theta(\omega), Z_{t, z}^{\nu}(\theta)(\omega) ; \nu\left(\omega^{\theta(\omega)}+\mathbf{T}_{\theta(\omega)}(\cdot)\right)\right)\right] d \mathbb{P}(\omega)
\end{aligned}
$$

where $\tilde{\omega} \in \Omega \mapsto \nu\left(\omega^{\theta(\omega)}+\mathbf{T}_{\theta(\omega)}(\tilde{\omega})\right)$ is viewed as a control in $\mathcal{U}_{\theta(\omega), Z_{t, z}^{\nu}(\theta)(\omega)}$ for fixed $\omega \in \Omega$. Since $J \leq V^{*}$ and $J(T, \cdot)=f$, it follows that

$$
\mathbb{E}\left[f\left(Z_{t, z}^{\nu}(T)\right)\right] \leq \mathbb{E}\left[V^{*}\left(\theta, Z_{t, z}^{\nu}(\theta)\right) \mathbf{1}_{\theta<T}+f\left(Z_{t, z}^{\nu}(T)\right) \mathbf{1}_{\theta=T}\right]
$$


and the result follows from the arbitrariness of $\nu \in \mathcal{U}_{t, z}$.

2. We now turn to the second inequality. It follows from Lemma A.2 in the Appendix that the set $\Gamma=\left\{(t, z, \nu) \in[0, T] \times \mathbb{R}^{d+1} \times \mathcal{U}: \nu \in \mathcal{U}_{t, z}\right\}$ is an analytic set. Clearly, $J$ is Borel measurable and therefore upper-semianalytic. It thus follows from Proposition 7.50 in [3] that, for each $\varepsilon>0$, we can find an analytically measurable map $\hat{\nu}^{\varepsilon}$ such that $J\left(t, z ; \hat{\nu}^{\varepsilon}(t, z)\right) \geq V(t, z)-\varepsilon$ and $\hat{\nu}^{\varepsilon}(t, z) \in \mathcal{U}_{t, z}$ on $D$. Since analytically measurable maps are also universally measurable, it follows from Lemma 7.27 in [3] that, for any probability measure $m$ on $[0, T] \times \mathbb{R}^{d+1}$, we can find a Borel measurable $\operatorname{map} \hat{\nu}_{m}^{\varepsilon}:(t, z) \in D \mapsto \mathcal{U}_{t, z}$ such that $J\left(t, z ; \hat{\nu}_{m}^{\varepsilon}(t, z)\right) \geq V(t, z)-\varepsilon \geq V_{*}(t, z)-\varepsilon$ for $m$-a.e. element of $D$. Let us now fix $\nu_{1} \in \mathcal{U}_{t_{0}, z_{0}}$ for some $\left(t_{0}, z_{0}\right) \in \operatorname{int}_{p}(D)$. Let $m$ be the measure induced by $\left(\theta, Z_{t_{0}, z_{0}}^{\nu_{1}}(\theta)\right)$ on $[0, T] \times \mathbb{R}^{d+1}$. By Theorem 2.1, $\left(\theta, Z_{t_{0}, z_{0}}^{\nu_{1}}(\theta)\right) \in D \mathbb{P}$ - a.s. so that

$$
\hat{\nu}_{m}^{\varepsilon}\left(\theta, Z_{t_{0}, z_{0}}^{\nu_{1}}(\theta)\right) \in \mathcal{U}_{\theta, Z_{t_{0}, z_{0}}^{\nu_{1}}(\theta)} \text { and } J\left(\theta, Z_{t_{0}, z_{0}}^{\nu_{1}}(\theta) ; \hat{\nu}_{m}^{\varepsilon}\left(\theta, Z_{t_{0}, z_{0}}^{\nu_{1}}(\theta)\right)\right) \geq V_{*}\left(\theta, Z_{t_{0}, z_{0}}^{\nu_{1}}(\theta)\right)-\varepsilon \mathbb{P}-\text { a.s. }
$$

Moreover, it follows from Lemma 2.1 of [21] that we can find $\nu_{2}^{\varepsilon} \in \mathcal{U}$ such that

$$
\nu_{2}^{\varepsilon} \mathbf{1}_{[\theta, T]}=\hat{\nu}_{m}^{\varepsilon}\left(\theta, Z_{t_{0}, z_{0}}^{\nu_{1}}(\theta)\right) \mathbf{1}_{[\theta, T]} d t \times d \mathbb{P}-\text { a.e. }
$$

This implies that $\nu^{\varepsilon}:=\nu_{1} \mathbf{1}_{\left[t_{0}, \theta\right)}+\nu_{2}^{\varepsilon} \mathbf{1}_{[\theta, T]} \in \mathcal{U}_{t_{0}, z_{0}}$ and

$\mathbb{E}\left[f\left(Z_{\theta, Z_{t_{0}, z_{0}}^{\nu_{1}}(\theta)}^{\varepsilon}(T)\right) \mid\left(\theta, Z_{t_{0}, z_{0}}^{\nu_{1}}(\theta)\right)\right]=J\left(\theta, Z_{t_{0}, z_{0}}^{\nu_{1}}(\theta) ; \hat{\nu}_{m}^{\varepsilon}\left(\theta, Z_{t_{0}, z_{0}}^{\nu_{1}}(\theta)\right)\right) \geq V_{*}\left(\theta, Z_{t_{0}, z_{0}}^{\nu_{1}}(\theta)\right)-\varepsilon \mathbb{P}-$ a.s.

and therefore

$$
\begin{aligned}
V\left(t_{0}, z_{0}\right) & \geq \mathbb{E}\left[\mathbb{E}\left[f\left(Z_{\theta, Z_{t_{0}, z_{0}}^{\nu_{1}}(\theta)}^{\nu_{1}}(T)\right) \mid\left(\theta, Z_{t_{0}, z_{0}}^{\nu_{1}}(\theta)\right)\right]\right] \\
& \geq \mathbb{E}\left[V_{*}\left(\theta, Z_{t_{0}, z_{0}}^{\nu_{1}}(\theta)\right) \mathbf{1}_{\theta<T}+f\left(Z_{t_{0}, z_{0}}^{\nu_{1}}(T)\right) \mathbf{1}_{\theta=T}\right]-\varepsilon .
\end{aligned}
$$

The required result then follows from the arbitrariness of $\nu_{1} \in \mathcal{U}_{t_{0}, z_{0}}$ and $\varepsilon>0$.

\subsection{The Hamilton-Jacobi-Bellman equation in the general case}

The proof of Theorem 3.1 follows from rather standard arguments based on Theorem 6.1 except that we have to handle the stochastic target constraint.

\subsubsection{Supersolution property for $t<T$}

Proof. [Proof of Eq. (3.7)] Let $\varphi$ be a smooth function such that $\left(t_{0}, z_{0}\right) \in \operatorname{int}_{p}(D) \cup \partial_{Z} D$ achieves a strict minimum (equal to 0 ) of $V_{*}-\varphi$ on $\operatorname{cl}(D)$. We also consider $\phi \in \mathcal{W}^{*}\left(t_{0}, x_{0}\right)$.

Case 1: We first consider the case where $\left(t_{0}, z_{0}\right) \in \operatorname{int}_{p}(D)$. We argue by contradiction and assume that

$$
\left(-\partial_{t} \varphi+H^{*} \varphi\right)\left(t_{0}, z_{0}\right)<0
$$

Since the coefficients $\mu_{Z}$ and $\sigma_{Z}$ are continuous, we can find an open bounded neighborhood $B \subset$ $\operatorname{int}_{p}(D)$ of $\left(t_{0}, z_{0}\right)$ and $\hat{u} \in U$ such that

$$
-\mathcal{L}_{Z}^{\hat{u}} \varphi \leq 0 \text { on } B .
$$


Let $\left(t_{n}, z_{n}\right)_{n}$ be a sequence in $B$ such that $V\left(t_{n}, z_{n}\right) \rightarrow V_{*}\left(t_{0}, z_{0}\right)$ and $\left(t_{n}, z_{n}\right) \rightarrow\left(t_{0}, z_{0}\right)$. Denote $\hat{Z}^{n}:=Z_{t_{n}, z_{n}}^{\hat{u}}$ where $\hat{u}$ is viewed as a constant control in $\mathcal{U}^{t_{n}}$ and let $\theta_{n}$ denote the first exit time of $\left(s, \hat{Z}^{n}(s)\right)_{s \geq t_{n}}$ from $B$. Since $\left(\theta_{n}, \hat{Z}^{n}\left(\theta_{n}\right)\right) \in B \subset \operatorname{int}_{p}(D)$, it follows from Theorem 2.1 that there exists a control $\nu^{n} \in \mathcal{U}_{t_{n}, z_{n}}$ such that $\nu^{n}=u$ on $\left[t_{n}, \theta_{n}\right)$. We now set $Z^{n}=\left(X^{n}, Y^{n}\right):=Z_{t_{n}, z_{n}}^{\nu^{n}}$ and observe that $Z^{n}=\hat{Z}^{n}$ on $\left[t_{n}, \theta_{n}\right]$ by continuity of the paths of both processes. It then follows from Itô's Lemma and (6.2) that, for $n$ large enough,

$$
\varphi\left(t_{n}, z_{n}\right) \leq \mathbb{E}\left[\varphi\left(\theta_{n}, Z^{n}\left(\theta_{n}\right)\right)\right] \leq \mathbb{E}\left[V_{*}\left(\theta_{n}, Z^{n}\left(\theta_{n}\right)\right)\right]-\zeta
$$

where

$$
\zeta:=\min _{(t, z) \in \partial_{p} B}\left(V_{*}-\varphi\right)>0 .
$$

Since $(\varphi-V)\left(t_{n}, z_{n}\right) \rightarrow\left(\varphi-V_{*}\right)\left(t_{0}, z_{0}\right)=0$ as $n \rightarrow \infty$, this contradicts Theorem 6.1 for $n$ large enough.

Case 2: We now turn to the case where $\left(t_{0}, z_{0}\right) \in \partial_{Z} D$. As above, we argue by contradiction and assume that

$$
\limsup _{(t, z) \rightarrow\left(t_{0}, z_{0}\right), \gamma \downarrow 0} \inf _{u \in U_{0, \gamma}(t, z, \phi)}\left(-\mathcal{L}_{Z}^{u} \varphi(t, z)\right)<0,
$$

recall (3.4). Note that this implies that $\mathcal{N}_{0}(t, z, D \phi(t, x)) \neq \emptyset$ for $(t, z)$ in a neighborhood of $\left(t_{0}, z_{0}\right)$. Using Assumption 3.1, we then deduce that there exists an open neighborhood $\mathcal{O}$ of $\left(t_{0}, z_{0}\right)$ and a Lipschitz continuous map $\hat{u}$ on $\mathcal{O}$ such that for all $(t, z) \in \mathcal{O}$

$$
\left.\begin{array}{r}
-\mathcal{L}_{Z}^{\hat{u}(t, z)} \varphi(t, z) \leq 0 \\
\mu_{Y}(z, \hat{u}(t, z))-\mathcal{L}_{X}^{\hat{u}(t, z)} \phi(t, x)>0 \\
\hat{u}(t, z) \in \mathcal{N}_{0}(z, D \phi(t, x))
\end{array}\right\}
$$

Let $\left(t_{n}, z_{n}\right)_{n}$ be a sequence in $\mathcal{O} \cap \operatorname{int}_{p}(D)$ such that $V\left(t_{n}, z_{n}\right) \rightarrow V_{*}\left(t_{0}, z_{0}\right)$ and $\left(t_{n}, z_{n}\right) \rightarrow\left(t_{0}, z_{0}\right)$. Let $\hat{Z}^{n}=\left(\hat{X}^{n}, \hat{Y}^{n}\right)$ denote the solution of (1.3) for the Markovian control $\hat{\nu}^{n}$ associated to $\hat{u}$ and initial condition $z_{n}$ at time $t_{n}$. Clearly, $\hat{\nu}^{n} \in \mathcal{U}^{t_{n}}$. Let $\theta_{n}$ denote the first exit time of $\left(s, \hat{Z}^{n}(s)\right)_{s \geq t_{n}}$ from $\mathcal{O}$. It follows from Itô's Lemma and the two last inequalities in (6.3) that

$$
\hat{Y}^{n}\left(\theta_{n}\right) \geq \phi\left(\theta_{n}, \hat{X}^{n}\left(\theta_{n}\right)\right)+y_{n}-\phi\left(t_{n}, x_{n}\right) .
$$

Since $w-\phi$ achieves a strict maximum at $\left(t_{0}, x_{0}\right)$ and $(w-\phi)\left(t_{0}, x_{0}\right)=0$, we can find $\kappa>0$ such that $w-\phi \leq-\kappa$ on $\partial \mathcal{O}$. Since, by definition of $\theta_{n}, \hat{Z}^{n}\left(\theta_{n}\right) \in \partial \mathcal{O}$, it follows from the previous inequality that

$$
\hat{Y}^{n}\left(\theta_{n}\right) \geq w\left(\theta_{n}, \hat{X}^{n}\left(\theta_{n}\right)\right)+\kappa+y_{n}-\phi\left(t_{n}, x_{n}\right)
$$

Using the fact that $y_{n}-\phi\left(t_{n}, x_{n}\right) \rightarrow y_{0}-w\left(t_{0}, x_{0}\right)=0$, recall (2.5), we thus deduce that, for $n$ large enough,

$$
\hat{Y}^{n}\left(\theta_{n}\right)>w\left(\theta_{n}, \hat{X}^{n}\left(\theta_{n}\right)\right) .
$$


In view of Corollary 2.1, we can then find a control $\nu^{n} \in \mathcal{U}_{t_{n}, z_{n}}$ such that $\nu^{n}=\hat{\nu}^{n}$ on $\left[t_{n}, \theta_{n}\right)$. Let us set $Z^{n}=\left(X^{n}, Y^{n}\right):=Z_{t_{n}, z_{n}}^{\nu^{n}}$ and observe that on $Z^{n}=\hat{Z}^{n}$ on $\left[t_{n}, \theta_{n}\right]$ by continuity of the paths of both processes. We can now appeal to the first inequality in (6.3) to obtain by the same arguments as in Case 1 above that

$$
V\left(t_{n}, z_{n}\right)<\mathbb{E}\left[V_{*}\left(\theta_{n}, Z^{n}\left(\theta_{n}\right)\right)\right]
$$

for $n$ large enough. Since $\nu^{n} \in \mathcal{U}_{t_{n}, z_{n}}$, this contradicts Theorem 6.1.

Remark 6.1. The relaxation in (3.4) is used in the above proof to ensure that (6.3) holds on a neighborhood of $\left(t_{0}, x_{0}\right)$. In the case where the function $w$ is smooth and the assumptions of Theorem 3.2 hold, it is not required anymore and we can replace of $F^{\phi *}$ by $F_{0,0}^{w}$ in the supersolution property of Theorem 3.1. Indeed, in this case,

$$
-\partial_{t} \varphi\left(t_{0}, x_{0}, w\left(t_{0}, x_{0}\right)\right)+F_{0,0}^{w} \varphi\left(t_{0}, x_{0}, w\left(t_{0}, x_{0}\right)\right)<0
$$

implies that

$$
-\mathcal{L}_{Z}^{\hat{u}\left(t_{0}, x_{0}\right)} \varphi\left(t_{0}, x_{0}, w\left(t_{0}, x_{0}\right)\right)<0
$$

where $\hat{u}(t, x):=\check{u}(x, w(t, x), D w(t, x))$ by Assumption 3.3, where, by Theorem 3.2,

$$
\mu_{Y}(x, w(t, x), \hat{u}(t, x))-\mathcal{L}_{X}^{\hat{u}(t, x)} w(t, x) \geq 0 \quad \text { and } \hat{u}(t, x) \in \mathcal{N}_{0}(x, w(t, x), D w(t, x)) .
$$

Since $\check{u}$ is Locally Lipschitz, it follows that

$$
-\mathcal{L}_{Z}^{\hat{u}(t, x)} \varphi(t, x, y)<0
$$

on a neighborhood of $\left(t_{0}, x_{0}, y_{0}\right)$. Now observe that (6.4), Itô's Lemma, the fact that $y_{n} \geq w\left(t_{n}, x_{n}\right)$ and a standard comparison result for SDEs show that

$$
Y_{t_{n}, x_{n}, y_{n}}^{\hat{\nu}^{n}} \geq w\left(\cdot, X_{t_{n}, x_{n}}^{\hat{\nu}^{n}}\right) \quad \text { on }\left[t_{n}, T\right]
$$

were $\hat{\nu}^{n}$ is the Markovian control associated to $\hat{u}$ and initial conditions $\left(t_{n}, x_{n}\right)$, i.e. defined by $\hat{\nu}_{t}^{n}=\hat{u}\left(t, X_{t_{n}, x_{n}}^{\hat{\nu}^{n}}(t)\right)$ for $t \geq t_{n}$. In view of our Standing assumption $g(\cdot, w(T, \cdot)) \geq 0$, this shows that $\hat{\nu}^{n} \in \mathcal{U}_{t_{n}, x_{n}, y_{n}}$, recall that $g$ is non-decreasing with respect to its second argument. Using (6.5) instead of the first inequality in (6.3) then allows to show by the same arguments as in the above proof that

$$
V\left(t_{n}, z_{n}\right)<\mathbb{E}\left[V_{*}\left(\theta_{n}, Z^{n}\left(\theta_{n}\right)\right)\right]
$$

for some well chosen stopping time $\theta_{n}$ and $n$ large enough. 


\subsubsection{Subsolution property for $t<T$}

Proof. [Proof of Eq. (3.9)] Let $\varphi$ be a smooth function such that $\left(t_{0}, z_{0}\right) \in \operatorname{int}_{p}(D) \cup \partial_{Z} D$ achieves a strict maximum (equal to 0 ) of $V^{*}-\varphi$ on $\operatorname{cl}(D)$. We argue by contradiction and assume that the subsolution property does not hold at $\left(t_{0}, z_{0}\right)$ for $\varphi$, i.e.

$$
-\partial_{t} \varphi\left(t_{0}, z_{0}\right)+H_{*} \varphi\left(t_{0}, z_{0}\right)>0 .
$$

Since the coefficients $\mu_{Z}$ and $\sigma_{Z}$ are continuous, we can then find an open bounded neighborhood $\mathcal{O}$ of the form $\left(t_{0}-\iota, t_{0}+\iota\right) \times O$ of $\left(t_{0}, z_{0}\right)$ such that $\mathcal{O} \cap \partial_{T} D=\emptyset$ and for all $u \in U$

$$
-\mathcal{L}_{Z}^{u} \varphi(t, z) \geq 0 \text { on } \mathcal{O}
$$

Let $\left(t_{n}, z_{n}\right)_{n}$ be a sequence in $\mathcal{O} \cap \operatorname{int}_{p}(D)$ such that $V\left(t_{n}, z_{n}\right) \rightarrow V^{*}\left(t_{0}, z_{0}\right)$ and $\left(t_{n}, z_{n}\right) \rightarrow\left(t_{0}, z_{0}\right)$. For each $n$, since $\left(t_{n}, z_{n}\right) \in D$, there exists a control $\nu^{n} \in \mathcal{U}_{t_{n}, z_{n}}$. Hence we set $Z^{n}:=Z_{t_{n}, z_{n}}^{\nu^{n}}$ and we let $\theta_{n}$ denote the first exit time of $\left(s, Z^{n}(s)\right)_{s \geq t_{n}}$ from $\mathcal{O} \cap\left(\operatorname{int}_{p}(D) \cup \partial_{Z} D\right)$. Note that, by definition of $\mathcal{U}_{t_{n}, z_{n}}$ and Theorem 2.1, $\left(s, Z^{n}(s)\right) \in \operatorname{int}_{p}(D) \cup \partial_{Z} D$ on $\left[t_{n}, T\right)$ and therefore $\left(\theta_{n}, Z^{n}\left(\theta_{n}\right)\right) \in \partial_{p} \mathcal{O}$. Applying Itô's Lemma and using (6.6), we then obtain

$$
\varphi\left(t_{n}, z_{n}\right) \geq \mathbb{E}\left[\varphi\left(\theta_{n}, Z^{n}\left(\theta_{n}\right)\right)\right] \geq \mathbb{E}\left[V^{*}\left(\theta_{n}, Z^{n}\left(\theta_{n}\right)\right)\right]+\zeta
$$

where, since $\left(t_{0}, z_{0}\right)$ achieves a strict maximum,

$$
-\zeta:=\max _{(t, z) \in \partial_{p} \mathcal{O}}\left(V^{*}-\varphi\right)<0 .
$$

Since $(\varphi-V)\left(t_{n}, z_{n}\right) \rightarrow\left(\varphi-V^{*}\right)\left(t_{0}, z_{0}\right)=0$ as $n \rightarrow \infty$, this contradicts Theorem 6.1 for $n$ large enough.

\subsubsection{Terminal condition}

The proof of the boundary condition at $t=T$ follows from the preceding arguments and the usual trick of perturbing the test function by adding a term of the form $\pm \sqrt{T-t}$. We only prove the supersolution property for $z_{0}=\left(x_{0}, y_{0}\right)$ such that $y_{0}>w\left(T, x_{0}\right)$. The subsolution property is proved similarly by combining the arguments below with the arguments of Section 6.2.2; it turns out that it is easier to handle.

Proof. [Proof of Eq. (3.8)] Fix $z_{0}:=\left(x_{0}, y_{0}\right)$ satisfying $y_{0}>w\left(T, x_{0}\right)$ and $\varphi$ be a smooth function such that $\left(T, z_{0}\right)$ achieves a strict minimum (equal to 0 ) of $V_{*}-\varphi$ on $\operatorname{cl}(D)$ and such that $H^{*} \varphi\left(T, z_{0}\right)<\infty$.

We argue by contradiction and assume that $V_{*}\left(T, z_{0}\right)<f_{*}\left(z_{0}\right)$. It follows that we can find $r, \eta>0$ such that

$$
\varphi \leq f_{*}-\eta \text { on }\left(\{T\} \times B_{r}\left(z_{0}\right)\right) \cap \operatorname{cl}(D)
$$

Let $\left(t_{n}, z_{n}\right)_{n}$ be a sequence $\operatorname{in}_{\operatorname{int}_{p}}(D)$ such that $V\left(t_{n}, z_{n}\right) \rightarrow V_{*}\left(T, z_{0}\right)$ and $\left(t_{n}, z_{n}\right) \rightarrow\left(T, z_{0}\right)$. As usual we modify the test function and introduce $\tilde{\varphi}:(t, z) \mapsto \varphi(z)-(T-t)^{\frac{1}{2}}$, so that 
$\left(T, z_{0}\right)$ is also a strict minimum point of $V_{*}-\tilde{\varphi}$. Since $H^{*} \varphi\left(T, z_{0}\right)<\infty$ by assumption and $-\partial_{t} \tilde{\varphi}(t, z)=-\partial_{t} \varphi(t, z)-\frac{1}{2}(T-t)^{-\frac{1}{2}}$, where $(T-t)^{-\frac{1}{2}} \rightarrow \infty$ as $t \rightarrow T$, we can choose $r>0$ small enough and $\hat{u} \in U$ such that

$$
-\mathcal{L}_{Z}^{\hat{u}} \tilde{\varphi} \leq 0 \text { on } \mathcal{V}_{0}:=[T-r, T) \times B_{r}\left(z_{0}\right)
$$

Since $w$ is continuous and $y_{0}>w\left(T, x_{0}\right)$, we can choose $r>0$ small enough so that

$$
\operatorname{cl}\left(\mathcal{V}_{0}\right) \subset\{(t, z) \in D: y \geq w(t, x)+r / 2\} \subset D
$$

Set $\hat{Z}^{n}:=Z_{t_{n}, z_{n}}^{\hat{u}}$ where $\hat{u}$ is viewed as a constant control in $\mathcal{U}^{t_{n}}$ and let $\theta_{n}$ denote the first exit time of $\left(s, \hat{Z}^{n}(s)\right)_{s \geq t_{n}}$ from $\mathcal{V}_{0}$. Since $\left(\theta_{n}, \hat{Z}^{n}\left(\theta_{n}\right)\right) \in \partial \mathcal{V}_{0} \subset D$, it follows from Theorem 2.1 that there exists a control $\nu^{n} \in \mathcal{U}_{t_{n}, z_{n}}$ such that $\nu^{n}=\hat{u}$ on $\left[t_{n}, \theta_{n}\right)$. Using (6.8), we then deduce that

$$
\tilde{\varphi}\left(t_{n}, z_{n}\right) \leq \mathbb{E}\left[\tilde{\varphi}\left(\theta_{n}, Z^{n}\left(\theta_{n}\right)\right)\right]
$$

where $Z^{n}:=Z_{t_{n}, z_{n}}^{\nu^{n}}$. Since $\left(T, z_{0}\right)$ is a strict minimum point for $V_{*}-\tilde{\varphi}$ and $\left(V_{*}-\tilde{\varphi}\right)\left(T, z_{0}\right)=0$, we can then find $\zeta>0$ such that $V_{*}-\zeta \geq \tilde{\varphi}$ on $\left\{(t, z) \in \partial \mathcal{V}_{0}: t<T\right\}$. Recalling (6.7) and the previous inequality, it follows that

$$
\tilde{\varphi}\left(t_{n}, z_{n}\right) \leq \mathbb{E}\left[\left(f_{*}\left(Z^{n}\left(\theta_{n}\right)\right)-\eta\right) \mathbf{1}_{\left\{\theta_{n}=T\right\}}+\left(V_{*}\left(\theta_{n}, Z^{n}\left(\theta_{n}\right)\right)-\zeta\right) \mathbf{1}_{\left\{\theta_{n}<T\right\}}\right] .
$$

Since $\nu^{n} \in \mathcal{U}_{t_{n}, z_{n}}$ and $\tilde{\varphi}\left(t_{n}, z_{n}\right)-V\left(t_{n}, z_{n}\right) \rightarrow 0$ as $n \rightarrow \infty$, the above inequality contradicts Theorem 6.1 for $n$ large enough.

\subsection{Subsolution property in the continuous boundary case}

In this section, we provide the proof of Proposition 3.1. We divide it in several Steps. From now on, we let $\varphi$ be a smooth function and $\left(t_{0}, z_{0}\right) \in \partial_{Z} D$ be such that $\left(t_{0}, z_{0}\right)$ is a strict local maximum point of $v-\varphi$, where $v$ is as in Proposition 3.1. We also fix $\phi \in \mathcal{W}_{*}\left(t_{0}, x_{0}\right)$ where $\left(x_{0}, y_{0}\right)=z_{0}$.

Lemma 6.1. There exists $u \in \mathcal{N}_{0}\left(z_{0}, D \phi\left(t_{0}, x_{0}\right)\right)$ such that $-\mathcal{L}_{Z}^{u} \varphi\left(t_{0}, z_{0}\right) \leq 0$.

Proof. Given $\eta>0$, we define

$$
\varphi_{\eta}(t, z):=\varphi(t, z)+f_{\eta}(t, z)
$$

where, for $z=(x, y) \in \mathbb{R}^{d} \times \mathbb{R}$,

$$
f_{\eta}(t, z):=\frac{1}{\eta}(y-\phi(t, x))-\eta(y-\phi(t, x))^{2} .
$$

Observe that $y_{0}=w\left(t_{0}, x_{0}\right)=\phi\left(t_{0}, x_{0}\right)$ while $y \geq w(t, x) \geq \phi(t, x)$ on $\operatorname{int}_{p}(D) \cup \partial_{Z} D$, since $\left(t_{0}, z_{0}\right) \in \partial_{Z} D$ and $w$ is continuous on $[0, T) \times \mathbb{R}^{d}$, recall (2.4)-(2.5). Observe also that $f_{\eta}(t, z) \geq 0$ for $0 \leq y-\phi(t, x) \leq \frac{1}{\eta^{2}}$. 
It follows that $\left(t_{0}, z_{0}\right)$ is a strict local maximum point of $v-\varphi_{\eta}$ on a neighborhood of $\left(t_{0}, z_{0}\right)$ on which $0 \leq y-\phi(t, x) \leq \frac{1}{\eta^{2}}$. We then deduce from the subsolution property of $v$ that

$$
-\partial_{t} \varphi_{\eta}\left(t_{0}, z_{0}\right)+H_{*}\left(z_{0}, D \varphi_{\eta}\left(t_{0}, z_{0}\right), D^{2} \varphi_{\eta}\left(t_{0}, z_{0}\right)\right) \leq 0 .
$$

For all $\varepsilon>0$, we can then find $\left(z_{\varepsilon}, q_{\varepsilon}, A_{\varepsilon}\right) \in \operatorname{cl}(D) \times \mathbb{R}^{d+1} \times \mathbb{S}^{d+1}$ and $u_{\varepsilon}^{\eta} \in U$ such that

$$
\left|\left(z_{\varepsilon}, q_{\varepsilon}, A_{\varepsilon}\right)-\left(z_{0}, D \varphi_{\eta}\left(t_{0}, z_{0}\right), D^{2} \varphi_{\eta}\left(t_{0}, z_{0}\right)\right)\right| \leq \varepsilon
$$

and, recalling that $y_{0}=\phi\left(t_{0}, x_{0}\right)$,

$$
-\partial_{t} \varphi\left(t_{0}, z_{0}\right)+\frac{1}{\eta} \partial_{t} \phi\left(t_{0}, x_{0}\right)+H^{u_{\varepsilon}^{\eta}}\left(z_{\varepsilon}, q_{\varepsilon}, A_{\varepsilon}\right) \leq \varepsilon .
$$

Using (6.9), the definitions of $\varphi_{\eta}$ and $H^{u}$, we thus obtain

$$
\begin{aligned}
\left\{-\partial_{t} \varphi\left(t_{0}, z_{0}\right)-\right. & \left.\mu_{Z}\left(z_{\varepsilon}, u_{\varepsilon}^{\eta}\right) \cdot D \varphi\left(t_{0}, z_{0}\right)-\frac{1}{2} \operatorname{Tr}\left[\sigma_{Z} \sigma_{Z}^{\top}\left(z_{\varepsilon}, u_{\varepsilon}^{\eta}\right) D^{2} \varphi\left(t_{0}, z_{0}\right)\right]\right\}+\frac{1}{\eta} \partial_{t} \phi\left(t_{0}, x_{0}\right) \\
& +H^{u_{\varepsilon}^{\eta}}\left(z_{\varepsilon}, D f_{\eta}\left(t_{0}, z_{0}\right), D^{2} f_{\eta}\left(t_{0}, z_{0}\right)\right) \leq \varepsilon\left(1+\left|\mu_{Z}\left(z_{\varepsilon}, u_{\varepsilon}^{\eta}\right)\right|+\left|\sigma_{Z}\left(z_{\varepsilon}, u_{\varepsilon}^{\eta}\right)\right|^{2}\right) .
\end{aligned}
$$

Now straightforward computations show that

$$
\begin{aligned}
H^{u_{\varepsilon}^{\eta}}\left(z_{\varepsilon}, D f_{\eta}(\right. & \left.\left.t_{0}, z_{0}\right), D^{2} f_{\eta}\left(t_{0}, z_{0}\right)\right)=\eta\left(\sigma_{Y}\left(z_{\varepsilon}, u_{\varepsilon}^{\eta}\right)-\sigma_{X}^{\top}\left(x_{\varepsilon}, u_{\varepsilon}^{\eta}\right) D \phi\left(t_{0}, x_{0}\right)\right)^{2} \\
+ & \frac{1}{\eta}\left(\mu_{X}\left(x_{\varepsilon}, u_{\varepsilon}^{\eta}\right) \cdot D \phi\left(t_{0}, x_{0}\right)+\frac{1}{2}\left(\sigma_{X} \sigma_{X}^{\top}\right)\left(x_{\varepsilon}, u_{\varepsilon}^{\eta}\right) D^{2} \phi\left(t_{0}, x_{0}\right)-\mu_{Y}\left(z_{\varepsilon}, u_{\varepsilon}^{\eta}\right)\right)
\end{aligned}
$$

(recall that $y_{0}=\phi\left(t_{0}, x_{0}\right)$ ). We now use Assumption 3.2 in order to deduce that $\left(u_{\varepsilon}^{\eta}\right)_{\varepsilon \in\left(0, \varepsilon_{0}\right)}$ is bounded for $\varepsilon_{0}$ small enough. Indeed, either $U$ is bounded or the right hand side of (6.11) is controlled by the first term of $H^{u_{\varepsilon}^{\eta}}\left(z_{\varepsilon}, D f_{\eta}\left(t_{0}, z_{0}\right), D^{2} f_{\eta}\left(t_{0}, z_{0}\right)\right)$. We thus get a converging subsequence $u_{\varepsilon_{n}}^{\eta} \rightarrow u_{0}^{\eta}$ with $\varepsilon_{n} \rightarrow 0$. We then deduce from (6.11) and (6.12) the following inequality

$$
\begin{aligned}
-\mathcal{L}_{Z}^{u_{0}^{\eta}} \varphi\left(t_{0}, z_{0}\right) & +\frac{1}{\eta} \partial_{t} \phi\left(t_{0}, x_{0}\right)+\eta\left(\sigma_{Y}\left(z_{0}, u_{0}^{\eta}\right)-\sigma_{X}^{\top}\left(x_{0}, u_{0}^{\eta}\right) D \phi\left(t_{0}, x_{0}\right)\right)^{2} \\
& +\frac{1}{\eta}\left(\mu_{X}\left(x_{0}, u_{0}^{\eta}\right) \cdot D \phi\left(t_{0}, x_{0}\right)+\frac{1}{2}\left(\sigma_{X} \sigma_{X}^{\top}\right)\left(x_{0}, u_{0}^{\eta}\right) D^{2} \phi\left(t_{0}, x_{0}\right)-\mu_{Y}\left(z_{0}, u_{0}^{\eta}\right)\right) \leq 0 .
\end{aligned}
$$

We now use Assumption 3.2 and the continuity of the coefficients again to obtain the required result by sending $\eta \rightarrow \infty$.

Lemma 6.2. For all $u_{i} \in \mathcal{N}_{0}\left(z_{0}, D \phi\left(t_{0}, x_{0}\right)\right)$ and $\gamma>0$ there exists $u_{f} \in \mathcal{N}_{0}\left(z_{0}, D \phi\left(t_{0}, x_{0}\right)\right)$ such that

$$
-\mathcal{L}_{Z}^{u_{f}} \varphi\left(t_{0}, z_{0}\right)-\gamma\left[\mu_{Y}\left(z_{0}, u_{i}\right)-\mathcal{L}_{X}^{u_{i}} \phi\left(t_{0}, x_{0}\right)\right]^{-}\left(\mu_{Y}\left(z_{0}, u_{f}\right)-\mathcal{L}_{X}^{u_{f}} \phi\left(t_{0}, x_{0}\right)\right) \leq 0 .
$$

Proof. For $\gamma>0$, define $\varphi_{\gamma}(t, z):=\varphi(t, z)+\ell_{\gamma}(t, z)$ where, for $z=(x, y) \in \mathbb{R}^{d} \times \mathbb{R}$,

$$
\ell_{\gamma}(t, z):=\gamma\left[\mu_{Y}\left(z_{0}, u_{i}\right)-\mathcal{L}_{X}^{u_{i}} \phi\left(t_{0}, x_{0}\right)\right]^{-}(y-\phi(t, x)) .
$$

Since $\ell_{\gamma}(t, z) \geq 0$ when $y \geq \phi(t, x)$, we conclude that $v-\varphi_{\gamma}$ achieves a strict maximum point of $v-\varphi_{\gamma}$ on $\operatorname{cl}(D)$. We thus can apply Lemma 6.1 to $\varphi=\varphi_{\gamma}$ and get $u_{f} \in \mathcal{N}_{0}\left(z_{0}, D \phi\left(t_{0}, x_{0}\right)\right)$ such that $-\mathcal{L}_{Z}^{u_{f}} \varphi_{\gamma}\left(t_{0}, z_{0}\right) \leq 0$. Direct computations imply the required result. 
Proof. [Proof of Proposition 3.1] Given $\eta>0$, it follows from Lemmata 6.1 and 6.2 that we can find a sequence $\left(u_{k}^{\eta}\right)_{k}$ in $\mathcal{N}_{0}\left(z_{0}, D \phi\left(t_{0}, x_{0}\right)\right)$ such that for all $k \geq 1$

$$
-\mathcal{L}_{Z}^{u_{k+1}^{\eta}} \varphi\left(t_{0}, z_{0}\right)-\eta\left[\mu_{Y}\left(z_{0}, u_{k}^{\eta}\right)-\mathcal{L}_{X}^{u_{k}^{\eta}} \phi\left(t_{0}, x_{0}\right)\right]^{-}\left(\mu_{Y}\left(z_{0}, u_{k+1}^{\eta}\right)-\mathcal{L}_{X}^{u_{k+1}^{\eta}} \phi\left(t_{0}, x_{0}\right)\right) \leq 0 .
$$

Since $\mathcal{N}_{0}\left(z_{0}, D \phi\left(t_{0}, x_{0}\right)\right)$ is compact under Assumption 3.2 (see Remark 3.4), it follows from the continuity of the coefficients that, for all $\eta>0$, we can find $u_{\eta} \in \mathcal{N}_{0}\left(z_{0}, D \phi\left(t_{0}, x_{0}\right)\right)$ such that

$$
-\mathcal{L}_{Z}^{u_{\eta}} \varphi\left(t_{0}, z_{0}\right)+\eta\left(\left[\mu_{Y}\left(z_{0}, u_{\eta}\right)-\mathcal{L}_{X}^{u_{\eta}} \phi\left(t_{0}, x_{0}\right)\right]^{-}\right)^{2} \leq 0
$$

Sending $\eta \rightarrow \infty$ and using once again the fact that $\mathcal{N}_{0}\left(z_{0}, D \phi\left(t_{0}, x_{0}\right)\right)$ is compact leads to the required result.

\subsection{Boundary condition when $w$ is smooth}

In this section, we prove Proposition 3.2 .

\subsubsection{Supersolution property in the domain}

We assume that $\hat{u}_{0}:=\hat{u}\left(t_{0}, x_{0}\right) \in \operatorname{int}(U)$, recall Theorem 3.2. Let $\phi$ be a smooth function and $\left(t_{0}, x_{0}\right) \in[0, T) \times \mathbb{R}^{d}$ be a strict minimum point for $\mathcal{V}_{*}-\phi$ such that $\left(\mathcal{V}_{*}-\phi\right)\left(t_{0}, x_{0}\right)=0$. We can assume without loss of generality that $\phi$ has polynomial growth. Set $y_{0}:=w\left(t_{0}, x_{0}\right)$ and define $\varphi_{n}$ by $\varphi_{n}(t, x, y):=\phi(t, x)-n(y-w(t, x))-\psi(t, x, y)$ with $\psi(t, x, y):=\left|x-x_{0}\right|^{2 p}+\left|y-y_{0}\right|^{2 p}+\left|t-t_{0}\right|^{2}$, $n \geq 1$, for some $p$ to be chosen later on. Since $V$ and $w$ have polynomial growth, for $p \geq 2$ large enough, $V_{*}-\varphi_{n}$ admits a local minimum point $\left(t_{n}, z_{n}\right)$ on $\operatorname{cl}(D)$. We note $z_{n}=\left(x_{n}, y_{n}\right)$. Writing that

$$
\begin{aligned}
\left(\mathcal{V}_{*}-\phi\right)\left(t_{0}, x_{0}\right) & =\left(V_{*}-\varphi_{n}\right)\left(t_{0}, x_{0}, w\left(t_{0}, x_{0}\right)\right) \\
& \geq\left(V_{*}-\varphi_{n}\right)\left(t_{n}, z_{n}\right) \\
& =\left(V_{*}-\phi\right)\left(t_{n}, z_{n}\right)+n\left(y_{n}-w\left(t_{n}, x_{n}\right)\right)+\psi\left(t_{n}, x_{n}, y_{n}\right)
\end{aligned}
$$

we deduce from the growth condition on $V$ that, after possibly passing to a subsequence, $\left(t_{n}, x_{n}, y_{n}\right) \rightarrow$ $\left(t_{\infty}, x_{\infty}, y_{\infty}:=w\left(t_{\infty}, x_{\infty}\right)\right)$ for some $\left(t_{\infty}, x_{\infty}\right) \in[0, T] \times \mathbb{R}^{d}$. It follows that

$$
\begin{aligned}
\left(\mathcal{V}_{*}-\phi\right)\left(t_{0}, x_{0}\right) & \geq \limsup _{n \rightarrow \infty}\left(\left(V_{*}-\phi\right)\left(t_{n}, z_{n}\right)+n\left(y_{n}-w\left(t_{n}, x_{n}\right)\right)+\psi\left(t_{n}, x_{n}, y_{n}\right)\right) \\
& \geq \liminf _{n \rightarrow \infty}\left(V_{*}-\phi\right)\left(t_{n}, z_{n}\right)+\limsup _{n \rightarrow \infty}\left(n\left(y_{n}-w\left(t_{n}, x_{n}\right)\right)+\psi\left(t_{n}, x_{n}, y_{n}\right)\right) \\
& \geq\left(V_{*}-\phi\right)\left(t_{\infty}, x_{\infty}, w\left(t_{\infty}, x_{\infty}\right)\right)+\limsup _{n \rightarrow \infty} n\left(y_{n}-w\left(t_{n}, x_{n}\right)\right)+\psi\left(t_{\infty}, x_{\infty}, y_{\infty}\right) \\
& \geq\left(V_{*}-\phi\right)\left(t_{\infty}, x_{\infty}, w\left(t_{\infty}, x_{\infty}\right)\right)+\liminf _{n \rightarrow \infty} n\left(y_{n}-w\left(t_{n}, x_{n}\right)\right)+\psi\left(t_{\infty}, x_{\infty}, y_{\infty}\right) \\
& \geq\left(\mathcal{V}_{*}-\phi\right)\left(t_{\infty}, x_{\infty}\right) \geq\left(\mathcal{V}_{*}-\phi\right)\left(t_{0}, x_{0}\right)
\end{aligned}
$$

which implies that

$$
\left(t_{n}, x_{n}, y_{n}, V_{*}\left(t_{n}, z_{n}\right), n\left(y_{n}-w\left(t_{n}, x_{n}\right)\right)\right) \rightarrow\left(t_{0}, x_{0}, y_{0}, \phi\left(t_{0}, x_{0}\right), 0\right)
$$


In particular, $t_{n}<T$ for $n$ large enough. Moreover, it follows from Theorem 3.2 that $\mathcal{N}_{0}\left(x_{n}\right.$, $\left.w\left(t_{n}, x_{n}\right), D w\left(t_{n}, x_{n}\right)\right) \neq \emptyset$. Set $\hat{u}_{n}:=\hat{u}\left(t_{n}, x_{n}\right)$ and note that $\hat{u}_{n} \rightarrow \hat{u}_{0} \in \operatorname{int}(U)$, by Assumption 3.3. It follows that $\hat{u}_{n} \in \operatorname{int}(U)$, for $n$ large enough.

We have two cases:

1. If $y_{n}=w\left(t_{n}, x_{n}\right)$, then it follows from Remark 6.1 that

$$
-\mathcal{L}_{Z}^{\hat{u}_{n}} \varphi_{n}\left(t_{n}, x_{n}, y_{n}\right) \geq 0
$$

2. If $y_{n}>w\left(t_{n}, x_{n}\right)$, then $\left(t_{n}, x_{n}, y_{n}\right) \in \operatorname{int}_{p}(D)$ and it follows Theorem 3.1 that (6.14) holds too. In both case, we thus have, by replacing $\varphi_{n}$ in (6.14),

$$
-\mathcal{L}_{X}^{\hat{u}_{n}} \phi\left(t_{n}, x_{n}\right)+n\left(\mu_{Y}\left(x_{n}, y_{n}, \hat{u}_{n}\right)-\mathcal{L}_{X}^{\hat{u}_{n}} w\left(t_{n}, x_{n}\right)\right)+\mathcal{L}_{Z}^{\hat{u}_{n}} \psi\left(t_{n}, x_{n}, y_{n}\right) \geq 0 .
$$

Since $\mu_{Y}$ is Lipschitz continuous, $n\left(y_{n}-w\left(t_{n}, x_{n}\right)\right) \rightarrow 0$ and $\mathcal{L}_{Z}^{\hat{u}_{n}} \psi\left(t_{n}, x_{n}, y_{n}\right) \rightarrow 0$, by (6.13), this implies that

$$
-\mathcal{L}_{X}^{\hat{u}_{0}} \phi\left(t_{0}, x_{0}\right)+\liminf _{n \rightarrow \infty} n\left(\mu_{Y}\left(x_{n}, w\left(t_{n}, x_{n}\right), \hat{u}_{n}\right)-\mathcal{L}_{X}^{\hat{u}_{n}} w\left(t_{n}, x_{n}\right)\right) \geq 0 .
$$

Since $\hat{u}_{n} \in \operatorname{int}(U)$ for $n$ large enough, Theorem 3.2 implies that $\mu_{Y}\left(x_{n}, w\left(t_{n}, x_{n}\right), \hat{u}_{n}\right)-\mathcal{L}_{X}^{\hat{u}_{n}} w\left(t_{n}, x_{n}\right)=$ 0 which proves the required result.

\subsubsection{Subsolution property in the domain.}

Let $\phi$ be a smooth function, $\left(t_{0}, x_{0}\right) \in[0, T) \times \mathbb{R}^{d}$ be a global strict maximum point for $\mathcal{V}^{*}-\phi$. Given $\varepsilon>0$, define for $n \geq 1$ the function $\varphi_{n}$ by

$$
\varphi_{n}(t, x, y):=\phi(t, x)+\psi(t, x, y)+\varepsilon n(y-w(t, x))+\varepsilon n^{2}(y-w(t, x))\left(w(t, x)-y+n^{-1}\right)
$$

with $\psi(t, x, y):=\left|t-t_{0}\right|^{2}+\left|x-x_{0}\right|^{2 p}+\left|y-y_{0}\right|^{2 p}$. Let $\left(t_{n}, x_{n}, y_{n}\right)_{n}$ be a sequence such that, for each $n,\left(t_{n}, x_{n}, y_{n}\right)$ is a maximizer on $\left\{(t, x, y) \in \operatorname{cl}(D): y-w(t, x) \leq n^{-1}\right\}$ of $V^{*}-\varphi_{n}$. Arguing as in the previous section, one easily checks that, for $p \geq 2$ large enough and after possibly passing to a subsequence,

$$
\left(t_{n}, x_{n}, n\left(y_{n}-w\left(t_{n}, x_{n}\right)\right)\right) \rightarrow\left(t_{0}, x_{0}, 0\right)
$$

In particular, we can assume, after possibly passing to a subsequence, that $t_{n}<T$ and $y_{n}-$ $w\left(t_{n}, x_{n}\right)<n^{-1}$ for all $n \geq 1$. It then follows from Corollary 3.1 that, for each $n \geq 1$, we can find $u_{n} \in U$ such that

$$
n^{-1} \geq-\mathcal{L}_{Z}^{u_{n}} \varphi_{n}\left(t_{n}, x_{n}, y_{n}\right)
$$

which, by direct computations, implies

$$
\begin{aligned}
n^{-1} & \geq-\mathcal{L}_{X}^{u_{n}} \phi\left(t_{n}, x_{n}\right)-2 \varepsilon n^{2}\left(n^{-1}+w\left(t_{n}, x_{n}\right)-y_{n}\right)\left(\mu_{Y}\left(x_{n}, y_{n}, u_{n}\right)-\mathcal{L}_{X}^{u_{n}} w\left(t_{n}, x_{n}\right)\right) \\
& +\varepsilon n^{2}\left|N^{u_{n}}\left(x_{n}, y_{n}, D w\left(t_{n}, x_{n}\right)\right)\right|^{2}-\mathcal{L}_{Z}^{u_{n}} \psi\left(t_{n}, x_{n}, y_{n}\right) .
\end{aligned}
$$


Note that (6.15) implies that

$$
n^{2}\left(n^{-1}+w\left(t_{n}, x_{n}\right)-y_{n}\right)=O(n)
$$

Using (3.11) and the previous inequality, we then deduce that the sequence $\left(u_{n}\right)_{n}$ is bounded and therefore converges, after possibly passing to a subsequence, to some $u_{0} \in U$. It also follows that $n\left|N^{u_{n}}\left(x_{n}, y_{n}, D w\left(t_{n}, x_{n}\right)\right)\right|^{2}$ is bounded, so that $N^{u_{0}}\left(x_{0}, w\left(t_{0}, x_{0}\right), D w\left(t_{0}, x_{0}\right)\right)=\lim _{n} N^{u_{n}}\left(x_{n}, y_{n}\right.$, $\left.D w\left(t_{n}, x_{n}\right)\right)=0$, which by Assumption 3.3, leads to $u_{0}=\hat{u}\left(t_{0}, x_{0}\right)$. Moreover, it follows from (6.15)-(6.16)-(6.17), the Lipschitz continuity of the coefficients and the convergence $u_{n} \rightarrow \hat{u}\left(t_{0}, x_{0}\right)$ that

$$
\begin{aligned}
\alpha(n) & \geq-\mathcal{L}_{X}^{\hat{u}\left(t_{0}, x_{0}\right)} \phi\left(t_{0}, x_{0}\right)+2 \varepsilon n^{2}\left|n^{-1}+w\left(t_{n}, x_{n}\right)-y_{n}\right|\left(\mathcal{L}_{X}^{u_{n}} w\left(t_{n}, x_{n}\right)-\mu_{Y}\left(x_{n}, w\left(t_{n}, x_{n}\right), u_{n}\right)\right) \\
& +\varepsilon n^{2}\left|N^{u_{n}}\left(x_{n}, w\left(t_{n}, x_{n}\right), D w\left(t_{n}, x_{n}\right)\right)\right|^{2}
\end{aligned}
$$

where $\alpha(n) \rightarrow 0$ as $n \rightarrow \infty$. In view of Assumption 3.4 and (6.17), taking the limit as $n \rightarrow \infty$ leads to

$$
0 \geq-\mathcal{L}_{X}^{\hat{u}\left(t_{0}, x_{0}\right)} \phi\left(t_{0}, x_{0}\right)-\varepsilon C
$$

for some $C>0$, which, by arbitrariness of $\varepsilon>0$ proves the required result.

\subsubsection{Subsolution property on the boundary}

Fix $x_{0} \in \mathbb{R}^{d}$ and a smooth function $\varphi$ such that $x_{0}$ achieves a strict maximum of $\mathcal{V}^{*}(T, \cdot)-\varphi$ such that $\mathcal{V}^{*}\left(T, x_{0}\right)-\varphi\left(x_{0}\right)=0$. If $\mathcal{W}_{*}\left(T, x_{0}\right) \neq \emptyset$, we can find a smooth function $\phi$ such that $x_{0}$ reaches a local minimum of $w(T, \cdot)-\phi$ such that $w\left(T, x_{0}\right)-\phi\left(x_{0}\right)=0$. For $n \geq 1$, set $\tilde{\varphi}_{n}(z):=$ $\varphi(x)+n(y-\phi(x))+\psi(x, y)+n^{2}(y-\phi(x))\left(\phi(x)-y+n^{-1}\right)$ where $\psi(x, y):=\left|x-x_{0}\right|^{2 p}+\left|y-y_{0}\right|^{2 p}$ for some $p \geq 2$. Arguing as above, we obtain that, for $p \geq 2$ large enough, there exists a sequence of local maximizers $\left(\tilde{x}_{n}, \tilde{y}_{n}\right)_{n}$ of $V^{*}(T, \cdot)-\tilde{\varphi}_{n}$ such that

$$
\left(\tilde{x}_{n}, n\left(\tilde{y}_{n}-\phi\left(\tilde{x}_{n}\right)\right), V^{*}\left(T, \tilde{x}_{n}, \tilde{y}_{n}\right)\right) \rightarrow\left(x_{0}, 0, \mathcal{V}^{*}\left(T, x_{0}\right)\right)
$$

For all $u \in U$, we now compute that

$$
\begin{aligned}
-\mathcal{L}_{Z}^{u} \tilde{\varphi}_{n}\left(z_{n}\right) & =-\mathcal{L}_{X}^{u} \varphi\left(\tilde{x}_{n}\right)-2 n^{2}\left(n^{-1}+\phi\left(\tilde{x}_{n}\right)-\tilde{y}_{n}\right)\left(\mu_{Y}\left(\tilde{x}_{n}, \tilde{y}_{n}, u\right)-\mathcal{L}_{X}^{u} \phi\left(\tilde{x}_{n}\right)\right) \\
& +n^{2} \mid N^{u}\left(\tilde{x}_{n}, \tilde{y}_{n},\left.D \phi\left(\tilde{x}_{n}\right)\right|^{2}-\mathcal{L}_{Z}^{u} \psi\left(\tilde{x}_{n}, \tilde{y}_{n}\right) .\right.
\end{aligned}
$$

In view of (3.11), this implies that $H_{*} \tilde{\varphi}_{n}\left(z_{n}\right)>-\infty$. It then follows from Theorem 3.1 that $V^{*}\left(T, \tilde{x}_{n}, \tilde{y}_{n}\right) \leq f^{*}\left(\tilde{x}_{n}, \tilde{y}_{n}\right)$ for all $n$. Sending $n \rightarrow \infty$ and using (6.18) shows that $\mathcal{V}^{*}\left(T, x_{0}\right) \leq$ $f^{*}\left(x_{0}, \phi\left(x_{0}\right)\right)=f^{*}\left(x_{0}, w\left(T, x_{0}\right)\right)$, which concludes the proof.

\subsubsection{Supersolution property on the boundary}

Step 1 . We first show that for any smooth function $\varphi$ and $\left(T, z_{0}\right) \in[0, T) \times \mathbb{R}^{d} \times \mathbb{R}$, such that $y_{0}=$ $w\left(t_{0}, x_{0}\right)$, with $z_{0}=\left(x_{0}, y_{0}\right)$, which is a strict minimum point for $V_{*}-\varphi$ such that $\left(V_{*}-\varphi\right)\left(T, z_{0}\right)=0$, 
we have

$$
\varphi\left(T, z_{0}\right) \geq f_{*}\left(z_{0}\right) \quad \text { if } \limsup _{\left(t^{\prime}, x^{\prime}\right) \rightarrow\left(T, x_{0}\right), t^{\prime}<T}\left|\hat{u}\left(t^{\prime}, x^{\prime}\right)\right|<\infty
$$

where $\hat{u}:=\check{u}(\cdot, w, D w)$.

We argue by contradiction, and assume that $\varphi\left(T, z_{0}\right)<f_{*}\left(z_{0}\right)$. Then, there exists $\eta, r>0$ small enough so that

$$
\varphi \leq f_{*}-\eta \text { on }\{T\} \times B_{r}\left(z_{0}\right)
$$

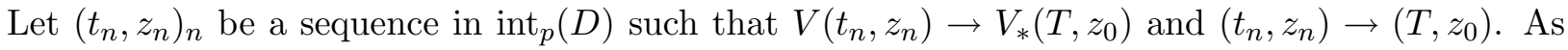
in Section 6.2.3, we modify the test function and introduce $\tilde{\varphi}:(t, z) \mapsto \varphi(z)-(T-t)^{\frac{1}{2}}$, so that $\left(T, z_{0}\right)$ is also a strict minimum point of $V_{*}-\tilde{\varphi}$. Recalling the right-hand side of (6.19) and

observing that $-\partial_{t} \tilde{\varphi}(t, z)=-\partial_{t} \varphi(t, z)-(T-t)^{-\frac{1}{2}}$, where $(T-t)^{-\frac{1}{2}} \rightarrow \infty$ as $t \rightarrow T$, we deduce that we can choose $r>0$ small enough such that

$$
-\mathcal{L}_{Z}^{\hat{u}} \tilde{\varphi}<0 \text { on } \mathcal{V}_{0}:=[T-r, T) \times B_{r}\left(z_{0}\right)
$$

In view of Theorem 3.2, $w$ satisfies

$$
\mu_{Y}(\cdot, w, \hat{u})-\mathcal{L}_{X}^{\hat{u}} w \geq 0 \text { on }[T-r, T) \times \mathbb{R}^{d} .
$$

Set $\hat{Z}^{n}:=Z_{t_{n}, z_{n}}^{\hat{u}^{n}}$ where $\hat{u}^{n}$ is the Markovian control associated to $\hat{u}$. Since $y_{n} \geq w\left(t_{n}, x_{n}\right)$ a standard comparison argument combined with (6.22) implies that $Y^{n}(T) \geq w\left(T, X^{n}(T)\right)$. Hence, $\hat{u}^{n} \in \mathcal{U}_{t_{n}, z_{n}}$. Moreover, (6.21) implies that

$$
\tilde{\varphi}\left(t_{n}, z_{n}\right) \leq \mathbb{E}\left[\tilde{\varphi}\left(\theta_{n}, \hat{Z}^{n}\left(\theta_{n}\right)\right)\right],
$$

where $\theta_{n}$ is the first exit time of $\left(s, \hat{Z}^{n}(s)\right)_{s \geq t_{n}}$ from $\mathcal{V}_{0}$. Since $\left(T, z_{0}\right)$ is a strict minimum point for $V_{*}-\tilde{\varphi}$ and $\left(V_{*}-\tilde{\varphi}\right)\left(T, z_{0}\right)=0$, we can then find $\zeta>0$ such that $V_{*}-\zeta \geq \tilde{\varphi}$ on $\left\{(t, z) \in \partial \mathcal{V}_{0}: t<T\right\}$. Recalling (6.20) and the previous inequality, it follows that

$$
\tilde{\varphi}\left(t_{n}, x_{n}, y_{n}\right) \leq \mathbb{E}\left[\left(f_{*}\left(\hat{Z}^{n}\left(\theta_{n}\right)\right)-\eta\right) \mathbf{1}_{\left\{\theta_{n}=T\right\}}+\left(V_{*}\left(\theta_{n}, \hat{Z}^{n}\left(\theta_{n}\right)\right)-\zeta\right) \mathbf{1}_{\left\{\theta_{n}<T\right\}}\right] .
$$

Since $\nu^{n} \in \mathcal{U}_{t_{n}, x_{n}, y_{n}}$ and $\tilde{\varphi}\left(t_{n}, z_{n}\right)-V\left(t_{n}, z_{n}\right) \rightarrow 0$ as $n \rightarrow \infty$, the above inequality contradicts Theorem 6.1 for $n$ large enough.

Step 2. The proof is concluded by arguing as in Section 6.4 .3 above and adapting the test function as in Section 6.4.1.

\section{Appendix: Dynamic programming for stochastic targets}

This Appendix is devoted to the proof of Theorem 2.1, which is essentially known from [21]. The only difference comes from the definition of the set of controls. Since the above arguments are almost the same as the one used in [21], we only insist on the differences and refer to this paper for some auxiliary results. We start with two important lemmata. 
Lemma A.1. The set $\left\{(t, z, \nu) \in[0, T] \times \mathbb{R}^{d+1} \times \mathcal{U}: \nu \in \mathcal{U}^{t}\right\}$ is closed in $[0, T] \times \mathbb{R}^{d+1} \times L^{2}([0, T] \times$ $\Omega)$ and the set $\Gamma:=\left\{(t, z, \nu) \in[0, T] \times \mathbb{R}^{d} \times \mathcal{U}: \nu \in \mathcal{U}_{t, z}\right\}$ is a Borel set in $[0, T] \times \mathbb{R}^{d+1} \times$ $L^{2}([0, T] \times \Omega)$.

Proof. Let $\left(t_{n}, \nu^{n}\right)_{n \geq 1}$ be a sequence in $[0, T] \times \mathcal{U}$ such that $\nu^{n} \in \mathcal{U}^{t_{n}}$ for each $n$, and $\left(t_{n}, \nu^{n}\right) \rightarrow$ $(t, \nu) \in[0, T] \times L^{2}([0, T] \times \Omega)$ strongly. Let $\xi$ be a continuous adapted bounded process. For $n \geq 1$, we have

$$
\mathbb{E}\left[\int_{0}^{T} \nu_{s}^{n} \xi_{s \wedge t_{n}} d s\right]=\int_{0}^{T} \mathbb{E}\left[\nu_{s}^{n}\right] \mathbb{E}\left[\xi_{s \wedge t_{n}}\right] d s
$$

since $\nu^{n}$ is independent on $\mathcal{F}_{t_{n}}$ and $\xi_{\cdot \wedge t_{n}}$ is $\mathcal{F}_{t_{n}}$-measurable. Sending $n \rightarrow \infty$ and using the continuity of $\xi$, we then obtain

$$
\mathbb{E}\left[\int_{0}^{T} \nu_{s} \xi_{s \wedge t} d s\right]=\int_{0}^{T} \mathbb{E}\left[\nu_{s}\right] \mathbb{E}\left[\xi_{s \wedge t}\right] d s
$$

By arbitrariness of $\xi$, this shows that $\nu$ is independent on $\mathcal{F}_{t}$. On the other hand, it follows exactly from the same arguments as in the proof of Lemma 3.1 of $[21]$ that $\left\{(t, z, \nu) \in[0, T] \times \mathbb{R}^{d+1} \times \mathcal{U}\right.$ : $g\left(Z_{t, z}^{\nu}(T)\right) \geq 0 \mathbb{P}-$ a.s. $\}$ is a Borel set. In view of the previous result, this implies that $\Gamma$ is the intersection of two Borel sets.

Lemma A.2. For any probability measure $m$ on $\left(D, \mathcal{B}_{D}\right)$, there exists a Borel measurable map $\phi_{m}:\left(D, \mathcal{B}_{D}\right) \rightarrow\left(\mathcal{U}, \mathcal{B}_{\mathcal{U}}\right)$ such that $\phi_{m}(t, z) \in \mathcal{U}_{t, z}$ for $m$-a.e. $(t, z) \in D$.

Proof. Appealing to Lemma A.1 above, the proof follows the same arguments as in the proof of Lemma 3.1 in [21].

Proof of Theorem 2.1. The first assertion follows from the same argument as in the proof of Theorem 3.1 in [21] by appealing to Lemma A.2 instead of Lemma 3.1 in [21]. We now prove the second one. Fix $(t, z) \in[0, T) \times \mathbb{R}^{d+1}, \theta$ a $[t, T]$-valued stopping time, $\nu \in \mathcal{U}^{t}$ and $\tilde{\nu} \in \mathcal{U}_{t, z}$ such that $\nu=\tilde{\nu}$ on $[t, \theta)$. Then, the flow property implies that

$$
\begin{aligned}
1 & =\mathbb{P}\left[g\left(Z_{\theta, Z_{t, z}^{\nu}(\theta)}^{\tilde{\nu}}(T)\right) \geq 0\right] \\
& =\iint \mathbf{1}_{\left\{g\left(Z_{\theta(\omega), Z_{t, z}^{\nu}(\theta)(\omega)}^{\tilde{\nu}(\omega)}(T)\left(\omega^{\theta(\omega)}+\mathbf{T}_{\theta(\omega)}(\tilde{\omega})\right)\right) \geq 0\right\}} d \mathbb{P}(\omega) d \mathbb{P}(\tilde{\omega}),
\end{aligned}
$$

where we use the notations $\omega^{r}:=\left(\omega_{t \wedge r}\right)_{t \leq T}$ and $\mathbf{T}_{r}(\omega):=\left(\omega_{s+r}-\omega_{r}\right)_{s \leq T-r}$ for $r \in[0, T]$. Viewing $\tilde{\omega} \in \Omega \mapsto \tilde{\nu}\left(\omega^{\theta(\omega)}+\mathbf{T}_{\theta(\omega)}(\tilde{\omega})\right)$ as a control independent on $\mathcal{F}_{\theta(\omega)}$ for fixed $\omega \in \Omega$, this shows that, for $\mathbb{P}$-a.e. $\omega \in \Omega,\left(\theta, Z_{t, z}^{\nu}(\theta)\right)(\omega) \in D$.

\section{References}

[1] J.-P. Aubin. Viability theory. Systems \& Control: Foundations \& Applications. Birkhäuser Boston Inc., Boston, MA, 1991. 
[2] G. Barles and J. Burdeau. The Dirichlet problem for semilinear second-order degenerate elliptic equations and applications to stochastic exit time control problems. Comm. Partial Differential Equations, 20(1-2):129-178, 1995.

[3] D. Bertsekas and S. E. Shreve. Stochastic Optimal Control: The Discrete Time Case, volume 139 of Mathematics in Science and Engineering. Academic Press, 1978.

[4] B. Bouchard. Stochastic targets with mixed diffusion processes. Stochastic Processes and their Applications, 101:273-302, 2002.

[5] B. Bouchard, R. Elie, and N. Touzi. Stochastic target problems with controlled loss. Technical report, CEREMADE, 2008.

[6] B. Bouchard and V. T. Nam. The American version of the geometric dynamic programming principle: Application to the pricing of american options under constraints. Technical report, CEREMADE, 2008.

[7] P. Boyle and W. Tian. Portfolio management with constraints. Mathematical Finance, $17(3): 319-343,2007$.

[8] M. Broadie, J. Cvitanic, and H. M. Soner. Optimal replication of contingent claims under portfolio constraints. Rev. Financ. Stud., 11:59-79, 1998.

[9] I. Capuzzo-Dolcetta and P.-L. Lions. Hamilton-Jacobi equations with state constraints. Trans. Amer. Math. Soc., 318(2):643-683, 1990.

[10] F. H. Clarke, Y. S. Ledyaev, R. J. Stern, and P. R. Wolenski. Nonsmooth analysis and control theory, volume 178 of Graduate Texts in Mathematics. Springer-Verlag, New York, 1998.

[11] M. Crandall, H. Ishii, and P.-L. Lions. User's guide to viscosity solutions of second order partial differential equations. American Mathemtical Society, 27:1-67, 1992.

[12] H. Föllmer and P. Leukert. Quantile hedging. Finance and Stochastics, 3(3):251-273, 1999.

[13] H. Ishii and S. Koike. A new formulation of state constraint problems for first-order PDEs. SIAM J. Control Optim., 34(2):554-571, 1996.

[14] H. Ishii and P. Loreti. A class of stochastic optimal control problems with state constraint. Indiana Univ. Math. J., 51(5):1167-1196, 2002.

[15] I. Karatzas and S. E. Shreve. Methods of Mathematical Finance. Springer-Verlag, 1998.

[16] N. E. Karoui, M. Jeanblanc, and V. Lacoste. Optimal portolio management with american capital garantee. J. Econ. Dyn. Control, 29:449-468, 2005.

[17] M. A. Katsoulakis. Viscosity solutions of second order fully nonlinear elliptic equations with state constraints. Indiana Univ. Math. J., 43(2):493-519, 1994. 
[18] J.-M. Lasry and P.-L. Lions. Nonlinear elliptic equations with singular boundary conditions and stochastic control with state constraints. I. The model problem. Math. Ann., 283(4):583630, 1989.

[19] N. Saintier. A general stochastic target problem with jump diffusion and an application to a hedging problem for large investors. Electronic Communications in Probability, 12, 2007.

[20] H. M. Soner. Optimal control with state-space constraint. I. SIAM J. Control Optim., 24(3):552-561, 1986.

[21] H. M. Soner and N. Touzi. Dynamic programming for stochastic target problems and geometric flows. Journal of the European Mathematical Society, 4:201-236, 2002.

[22] H. M. Soner and N. Touzi. Stochastic target problems, dynamic programming and viscosity solutions. SIAM Journal on Control and Optimization, 41:404-424, 2002. 